\title{
Die LandFrauenorganisationen und ihr lokales Engagement im Spiegel der Regionalentwicklung
}

\author{
Laura Suarsana ${ }^{1}$ iD
}

Eingegangen: 21. Dezember 2016 / Angenommen: 21. Juli 2017 / Online publiziert: 7. August 2017

(C) Springer-Verlag GmbH Deutschland 2017

Zusammenfassung Dieser Beitrag zeigt die gesellschaftliche Bedeutung der deutschen LandFrauenorganisationen als Element insbesondere der ländlichen Zivilgesellschaft in Deutschland. Er liefert auf der Basis qualitativer Experteninterviews und sekundären Materials einen Überblick über die LandFrauen als zivilgesellschaftliche Organisationen und als Untersuchungsgegenstand sozialwissenschaftlicher Forschung. Die LandFrauen sind mit rund 450.000 Mitgliedern und über 10.600 Ortsvereinen, die in Kreisverbänden, Landesverbänden und einem Bundesverband gebündelt sind, deutschlandweit vertreten. Sie setzen sich unter anderem für die Interessen von Frauen ein, sind wichtiger Träger von Erwachsenenbildung und wirken als organisatorischer Rahmen für Aktivitäten und bürgerschaftliches Engagement ihrer Mitglieder, das sich vor allem auf kommunaler Ebene entfaltet. Der Beitrag kommt zu dem Ergebnis, dass die LandFrauen zur lokalen sozialen und kulturellen Infrastruktur in ländlichen Räumen erheblich beitragen. Gleichzeitig sind sie Partnerinnen innerhalb koordinierter Regionalentwicklung und stehen in Relation zur lokalen Ökonomie. Abschließend werden Perspektiven einer weiterführenden wissenschaftlichen Betrachtung aufgezeigt, unter anderem in Hinblick auf die Einbettung von Engagement in zivilgesellschaftliche Organisationen, Geographien von Engagement, Regional Governance und öffentliche Güter. Damit legt der Beitrag die Grundlagen für weiterführende Forschung.

Laura Suarsana

suarsana@uni-heidelberg.de

1 Institut für Geographie, Universität Heidelberg, Berliner Straße 48, 69120 Heidelberg, Deutschland
Schlüsselwörter LandFrauen · Regionalentwicklung · Bürgerschaftliches Engagement · Zivilgesellschaft · Ländlicher Raum

\section{The German Country Women's Associations and their civic engagement in the light of regional development}

Abstract This article highlights the societal relevance of the German Country Women's Associations (LandFrauen) as an element of German civil society especially in rural regions. On the basis of qualitative expert interviews and secondary material, this article provides an overview of the LandFrauen as civil society organizations and as a subject of social scientific research. German Country Women's Associations are represented throughout Germany with approximately 450,000 members in over 10,600 local associations, district associations, regional associations and a federal association. They represent, among other activities, the interests of women, are important providers of adult education and act as an organizational framework for the activities and civic engagement of their members, particularly at local community level. The article concludes that the LandFrauen contribute significantly to the local social and cultural infrastructure in rural areas. Concurrently, they are partners within coordinated regional development activities and stand in relation to the local economy. Additionally, this article presents perspectives for further scientific exploration with regard to the embedding of civic engagement into civil society organizations, geographies of engagement, regional governance and public goods. Therefore, this contribution lays the foundation for further research. 
Keywords German Country Women's Associations · Regional development - Civic engagement - Civil society · Rural area

\section{LandFrauen ${ }^{1}$ als Element der Zivilgesellschaft im ländlichen Raum: Zielsetzung und Struktur dieses Beitrags}

Mit wachsender Kritik an der „Dominanz regionalökonomischer und wirtschaftspolitischer Lösungen “ für städtische und regionale Entwicklung (Moss/Gudermann 2009: 15) sind Zivilgesellschaft und bürgerschaftliches Engagement zunehmend in den Fokus von Wissenschaft, Politik und Praxis gerückt (Schulte 2015: 31). Insbesondere in ländlichen Räumen wird bürgerschaftlichem Engagement Bedeutung unter anderem als Träger von Infrastruktur, sozialer Angebote und der Daseinsvorsorge zugeschrieben (Naumann/ Reichert-Schick 2015; Steinführer 2015). Mit den LandFrauenvereinen und -verbänden wird im Folgenden eine nur wenig beforschte zivilgesellschaftliche Organisationsvielfalt vorgestellt, die in ländlichen, aber auch städtischen Regionen Frauen aller Berufs- und Altersgruppen einbezieht. In über 10.600 Ortsvereinen (dlv 2016: 38) nehmen rund 450.000 Mitglieder ${ }^{2}$ Bildungs- und Veranstaltungsangebote der LandFrauenvereine und -verbände wahr, setzen sich in diesem Rahmen für die Interessen von Frauen ein und engagieren sich innerhalb dieses organisational-institutionellen Kontextes für ihr lokales Umfeld.

Dieser Beitrag stellt die Frage nach der regionalen und gesellschaftlichen Bedeutung dieser Organisationen. Im Anschluss an die methodischen Darlegungen (Kapitel 2) liefert er erstens eine Einordnung der LandFrauenorganisationen als Untersuchungsgegenstand in die bisherige Forschung (Kapitel 3) und einen deskriptiven Überblick über die LandFrauen (Kapitel 4). Zweitens liefert er eine Reflexion der Bedeutung der LandFrauen in Hinblick auf regionalentwicklungsbezogene Fragen (Kapitel 5). Eingebettet in die wissenschaftliche Debatte um raum- und regionalentwicklungsbezogene Perspektiven auf bürgerschaftliches Engagement werden drittens Potenziale ihrer wissenschaftlichen Betrachtung aufgezeigt (Kapitel 6). Damit rahmt dieser Beitrag die LandFrauenorganisationen als Forschungsgegenstand und legt die Grundlage für eine weiterführende Forschungsagenda (Kapitel 7).

\footnotetext{
${ }^{1}$ Entsprechend der organisationalen Eigenbezeichnungen und in Abgrenzung von der Gesamtheit auf dem Lande lebender Frauen wird im Folgenden diese Schreibweise mit kapitalisiertem F verwendet.

2 Auskunft des Bundesverbands (Stand Oktober 2016); dies inkludiert zahlende Mitglieder sowie Ehren- und Fördermitglieder.
}

\section{Methodisches Vorgehen}

Die nachfolgenden Ausführungen basieren auf ersten Ergebnissen einer laufenden qualitativ-quantitativen Studie über die LandFrauenorganisationen. In die Ausarbeitung einbezogen wurden die Inhalte leitfadengestützter Experteninterviews, die im Zuge des explorativen Projektauftakts mit der Präsidentin des Bundesverbands, mit der Geschäftsführerin eines Landesverbands, mit einer Kreisgeschäftsführerin und einer Ortsvorsitzenden, die beide ebenfalls Mitglied im Landesvorstand sind, sowie mit einem Ortsvereinsmitglied geführt wurden (I 1-I 5). Ziel war, die Organisationen in ihrem Aufbau und ihrem Handlungsumfang strukturell zu erfassen. Die Auswahl der Interviewpartnerinnen erfolgte aufgrund ihrer Rolle innerhalb der LandFrauenorganisationen, es wurden damit Expertinnen aller Ebenen der LandFrauenarbeit einbezogen. Durch das Delegiertenprinzip (vgl. Kapitel 4.2) verfügen die Gesprächspartnerinnen über fundiertes Wissen sowohl über die Verbands- als auch über die Kreisverbands- und Ortsvereinsebene. Nach ersten Kontaktaufnahmen wurden weitere Gesprächspartnerinnen durch Empfehlungen akquiriert. Die Gespräche fanden zwischen Juni 2015 und Januar 2016 statt. Im Anschluss erfolgte ein weiterer inhaltlicher Austausch zur Verifizierung extrahierter Interviewinhalte. Die geführten Gespräche wurden vollständig transkribiert, computergestützt mittels der Software MAXQDA12 codiert und inhaltsanalytisch zusammenfassend (vgl. Mayring 2015: $69 \mathrm{f}$.) ausgewertet. Da eine sozialwissenschaftliche Aufarbeitung der LandFrauenorganisationen als Element der gegenwärtigen Zivilgesellschaft in Deutschland bislang aussteht, erscheint das qualitative Vorgehen als eine angemessene Möglichkeit, um das Forschungsfeld aufzuschließen. Dies impliziert die Herausforderung, dass die in Expertengesprächen gewonnenen Informationen individuell und subjektiv beeinflusst und konnotiert sind. Diese werden erst im Zuge der weiteren Forschung durch Methodentriangulation und theoretische Sättigung kontrolliert werden können.

Neben der Empirie erfolgt die Darstellung der LandFrauenorganisationen auf der Grundlage primärer und sekundärer Quellen. Als primäre Quellen werden Eigenveröffentlichungen und die Internetauftritte des Bundesverbands und der Landesverbände einbezogen. Sekundärliteratur über die LandFrauen liegt in nur begrenztem Rahmen vor. Der bisherige Forschungsstand dazu wird in Kapitel 3.2 umrissen. 


\section{Die LandFrauenorganisationen als Untersuchungsgegenstand}

\subsection{Einordnung in die deutsche Engagement-, Vereins- und Verbandslandschaft}

Die LandFrauen können als wichtiges Element der deutschen Zivilgesellschaft und Engagementlandschaft bezeichnet werden. Grundlegend für die Debatte um bürgerschaftliches Engagement in Deutschland ist die Definition der Enquete-Kommission „Zukunft des bürgerschaftlichen Engagements" (Klein/Schwalb 2014: 1). Demnach ist bürgerschaftliches Engagement freiwillig, ,nicht direkt auf persönlichen materiellen Gewinn ausgerichtet", gemeinwohlorientiert, es findet „im öffentlichen Raum“ abseits „,vom Markt", „dem staatlichen Bereich oder der Privatsphäre“ und in Gemeinschaft statt bzw. ist "gemeinschaftsbezogen“" (Deutscher Bundestag 2002: 38 f.). Die Zivilgesellschaft ${ }^{3}$ bzw. der „Dritte Sektor“ bildet dabei den „,institutionellen Kern oder die Infrastruktur" für bürgerschaftliches Engagement (Alscher/Priller 2011: 720). „Zwei Drittel dieses Engagements finden in organisationalen Kontexten statt" (Krimmer/Priemer 2013: 8). ${ }^{4}$ Vereine haben einen großen Anteil am „Dritten Sektor“ in Deutschland. Für Ende 2015 lag allein die Zahl registrierter Vereine bei knapp 600.000 (Bundesamt für Justiz 2016: 7). Zimmer (2007b: 88) hebt drei Funktionen von Vereinen und Vereinswesen hervor: erstens die des „Mediums der politischen Sozialisation und einer ,Schule der Demokratie““, zweitens die des „,vorpolitischen Raums und Forums der Meinungsbildung sowie die der Machtbasis für Politiker“ sowie drittens die des „Bindeglieds zwischen Mikro- und Makro-Ebene und integrativen Elementes für Staat und Gesellschaft". Verbände sind hinsichtlich ihrer Rechtsform den Vereinen gleich, sind aber „in der Regel [...] Dachorganisationen“, die auf verschiedenen geographischen Ebenen aktiv sind (Zimmer 2007a: 394). Primär werden ihnen die Funktionen der Interessenvertretung und -bündelung zugeschrieben. Zimmer (2007a: 396) verweist darüber hinaus auf Funktionen als „Produzenten von Gütern und Leistungen“, als Dienstleister sowie ,,über ihre lokal tätigen Mitgliedsorganisationen [auf] Funktionen der affirmativen Bindung" und sozial-integrative Funktionen.

\footnotetext{
${ }^{3}$ Nach Kockas bereichslogischer Definition umfasst diese den „,selbstorganisierten, dynamischen, spannungsreichen öffentlichen Raum der Vereine, Netzwerke, Bewegungen und Organisationen ,zwischen Staat, Wirtschaft und Privatsphäre“ (Kocka 2003: 32).

4 Das Engagement der LandFrauen erstreckt sich vom „Basisengagement" der Mitglieder für das lokale Umfeld (Schulte 2015: 115) bis zur „Zivilgesellschaftsvertretung“ im Sinne der Übernahme von Funktionen innerhalb der Organisationen (Schulte 2015: 116).
}

\subsection{Stand der Forschung}

Verschiedene Publikationen arbeiten den frühen Entstehungs- und Entwicklungsprozess der LandFrauenorganisationen auf (Schwarz 1990; Bridenthal 1994; Helmle 2009; Sawahn 2009; Sawahn 2012). Daneben werden die LandFrauenorganisationen als Element der Verbandslandschaft in Deutschland diskutiert und in Untersuchungen von Frauen- und Agrarverbänden bzw. Lobbyorganisationen einbezogen (z. B. Biegler 2001). Icken (2002: 9) konstatiert in ihrer Untersuchung des Deutschen Frauenrats, dass „Frauenorganisationen [...] in der öffentlichen Wahrnehmung der Bundesrepublik Deutschland anders als zum Beispiel Sportorganisationen oder gar wirtschaftsnahe Organisationen keine Rolle [spielten]“. Rudolph und Schirmer (2004: 145) verweisen auf die Bedeutung lokaler Frauennetzwerke für die Arbeit von kommunalen Frauen- und Gleichstellungsbeauftragten. Sie erläutern, dass zwischen lokalen sozialen Frauenprojekten der neuen Frauenbewegung und „traditionellen“ Frauenverbänden, in welche sie auch die LandFrauenverbände einordnen, aufgrund unterschiedlicher politischer Ausrichtung und Ziele lange kaum lokale Zusammenarbeit zustande kam, und heben hervor, dass „Orts- und Landesgruppen, die in nationalen Dachverbänden zusammengeschlossen sind“, „ihr politisches Handeln häufig eher im Zusammenhang ihres Gesamtverbandes verorten als im jeweiligen kommunalpolitischen Kontext" (Rudolph/Schirmer 2004: 162 f.). ${ }^{5}$

Verschiedene Arbeiten diskutieren die Bildungsarbeit der LandFrauenorganisationen in den Themenkontexten Erwachsenenbildung und lebenslanges Lernen (Kaschuba 1996; Baumgart/Becker/Borsch et al. 2004; Köhler/Gonon 2004). Im Hinblick auf lokale und regionale Entwicklungen präsentieren bzw. analysieren verschiedene Arbeiten regionale Projekte unter Beteiligung oder Trägerschaft der LandFrauenvereine/-verbände, unter anderem zu Bildungsthemen (Ambos/Greubel 2012), Erwerbsmöglichkeiten und Arbeitsplätzen im ländlichen Raum (Putzing 2003; Putzing 2005), Tourismus (Habermann 1995; Kistemann 2003) und Biodiversität (Lütt 2007).

Mit der Rolle von Landfrauen als ,treibende Kraft der Dorfentwicklung" setzte sich 2005 eine Tagung des Gesprächskreises Ländlicher Raum auseinander (RosaLuxemburg-Stiftung 2005). Dabei wurde das breite Mitgliederspektrum als Potenzial ,der wirtschaftlichen, sozialen und kulturellen Entwicklung auf dem Lande“ - mit entsprechend ausdifferenzierten Interessenlagen - hervorgehoben (Krambach 2005: 6) und auf die Bedeutung der LandFrauen als lokal erfahrene Multiplikatoren hingewie-

\footnotetext{
5 Inwieweit dies für die LandFrauen zutrifft, ist kritisch zu hinterfragen angesichts der hohen Eigenständigkeit und starken lokalen Einbettung der Ortsvereine (vgl. Kapitel 4).
} 
sen (Krambach 2005: 8). Koch (2012: 25) verweist auf die „Zielgruppenorganisation“ des LandFrauenverbands, der als Interessenvertretung der Frauen im ländlichen Raum am besten vertreten sei.

Bisherige Arbeiten haben sich entweder nur mit Teilaspekten der Rolle der LandFrauen im ländlichen Raum auseinandergesetzt oder ihre Arbeit lediglich im Kontext anderer Fragestellungen betrachtet. Es fehlt jedoch eine ganzheitliche Betrachtung ihrer Bedeutung für die Regionalentwicklung und den ländlichen Raum aus sozialwissenschaftlicher Perspektive. Die folgenden Ausführungen geben einen Überblick darüber, wie das Engagement der LandFrauen organisatorisch gerahmt ist, und stellen die Organisationen hinsichtlich ihrer Struktur, Historie und ihres Aktivitätsspektrums vor, um anschließend die regionale Rolle und Bedeutung der LandFrauen und Forschungspotenziale hinsichtlich ihrer Betrachtung zu reflektieren.

\section{Die LandFrauen als zivilgesellschaftliche Organisationen}

\subsection{Historische Entwicklung}

Der erste landwirtschaftliche Hausfrauenverein wurde am 2. Februar 1898 in der Kreisstadt Rastenburg gegründet und umfasste 15 Mitglieder (Sawahn 2009: 13). Zentrale Gründungsfigur war die ostpreußische Gutsfrau Elisabeth Boehm, die ,31 Jahre lang von der Orts- bis zur Reichsebene fungierte" (Sawahn 2009: 13) und damit anstrebte, „Frauen zum Zwecke ihrer ländlich-hauswirtschaftlichen und kulturellen Weiterbildung $\mathrm{zu}$ organisieren“ (Schwarz 1990: 47). Vorbilder waren die bürgerliche Frauenbewegung und die agrarischen Männerbünde (Sawahn 2009: 65). Die frühe Vereinsarbeit steht im historischen Kontext ,beginnender politischer, sozialer und kultureller Transformationsprozesse" sowie eines Wandels der agrarischen Produktion (Sawahn 2009: 25). Diese ersten Gründungen waren Vorläufer der heutigen LandFrauenvereine (dlv o.J.c). In den darauf folgenden 100 Jahren entwickelte sich die LandFrauenbewegung als Interessenvertretung der deutschen Landfrauen ,über drei politische Systeme hinweg - Monarchie, Republik, Diktatur und wieder Republik“ (Sawahn 2009: 14). ${ }^{6} 1947$ wurde der Bundesverband gegründet. Nach der Wiedervereinigung gründeten sich auch in den neuen Bundesländern LandFrauenvereine (dlv o.J.c). Unterstützend waren dabei bestehende Kontakte westdeutscher Landfrauen ,zu den Landfrauenkommissionen der Landesbauernverbände in der DDR“ (Icken 2002: 178), sodass sich ,in jedem Bundesland [...] ein gut funktionierender Landesverband“

\footnotetext{
${ }^{6}$ Vgl. Schwarz (1990), Sawahn (2009).
}

herausbilden konnte (Icken 2002: 186). Aufgrund des historischen Prozesses ihrer Entstehung bestehen aktuell 22 Landesverbände. In den westlichen Bundesländern sind die LandFrauen deutlich stärker vertreten (vgl. Abbildung 1) (I 2; I 3).

\subsection{Organisationsstruktur, Finanzierung und Mitgliederspektrum}

Die Organisation der LandFrauen erstreckt sich über vier Ebenen: Die rund 10.600 Ortsvereine bilden die unterste Ebene der LandFrauenorganisationen. Innerhalb dieser vollzieht sich ein Großteil der Mitgliederaktivitäten. Die lokalen Vereine sind in 422 Kreisverbänden, 22 Landesverbänden und dem Bundesverband organisiert (vgl. Abbildung 2; dlv 2016: 38). Dabei variiert die konkrete Ausgestaltung der lokalen Vereine je nach Landesverband (I 1).

Die Landesverbände sind als gemeinnützige und registrierte Vereine überwiegend eigenständige Organisationen. Ausnahmen bilden die LandFrauenverbände Südbaden, Württemberg-Hohenzollern und Rheinland-Nassau sowie die LandFrauengruppe im Bayerischen Bauernverband. Diese sind in die jeweiligen Landesbauernverbände eingegliedert und unterliegen damit deren Satzung. Einhergehend weisen sie auch abweichende Organisations- und teils Mitgliederstrukturen auf, die sich an der Struktur der Bauernverbände orientieren. Innerhalb der Landesverbände sind die Orts- und Kreisverbände oft ebenfalls registrierte Vereine mit eigener Satzung, in einigen Verbänden teilen sie als nicht eingetragene Vereine die Satzung des Landesverbandes (I 1; I 2; I 3).

Die Aufgaben der Ortsvereine werden auf der regionalen Ebene durch die Kreisverbände unterstützt und gebündelt. Aus den Orts- und Kreisverbänden werden Delegierte an den Landesverband entsandt. Diese treffen in der Mitglieder- oder Delegiertenversammlung die Entscheidungen für den Landesverband und wählen das Präsidium bzw. den Vorstand. Weiteres Präsidiumsmitglied ist zumeist die Landesgeschäftsführerin als beratende Stimme, teilweise werden Vorstände des Landesbauern- oder Landjugendverbands als weitere Mitglieder benannt. Die Vereins- und Verbandsvorstände sowie die Delegierten sind ehrenamtlich tätig. Daneben werden die Landes-, teils auch große Kreisverbände, durch eine hauptamtliche Geschäftsführerin und weitere Geschäftsstellenmitarbeiter unterstützt. Daneben sind in einige Landesverbände noch weitere Organisationen integriert. In Baden-Württemberg besitzen die drei Landesverbände eigene Bildungs- und Sozialwerke, die als Träger der ländlichen Erwachsenenbildung anerkannt sind und deren Projekte und Qualifizierungen im Bereich „Innovative Maßnahmen für Frauen im ländlichen Raum“ 


\section{LandFrauenverbände in Deutschland 2015}

\section{Anzahl Veranstaltungen}

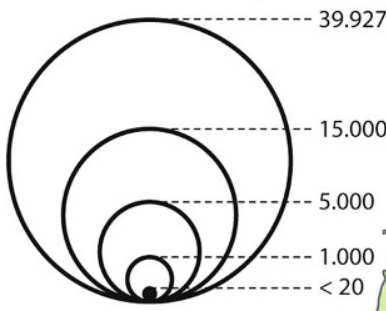

Insgesamt: 108.216

Anzahl Ortsvereine/

Mitglieder

zahlende Mitglieder

Ortsvereine

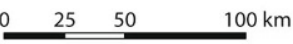

Kartographie: U. Selgert, V. Schniepp Kartengrundlage: $\odot$ BKG (2015)
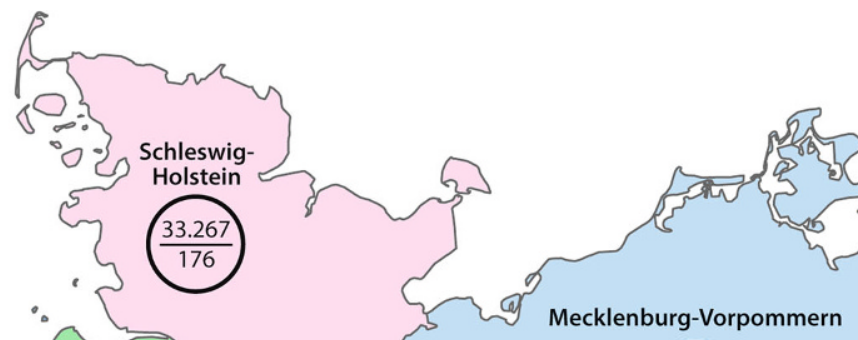

Mecklenburg-Vorpommern
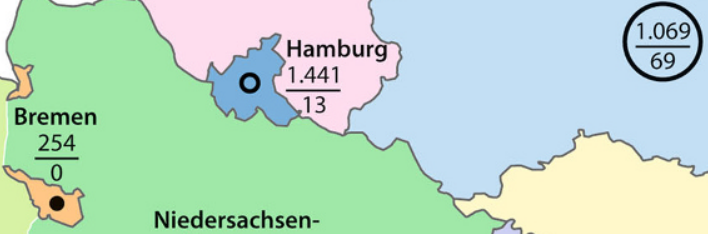

Niedersachsen-
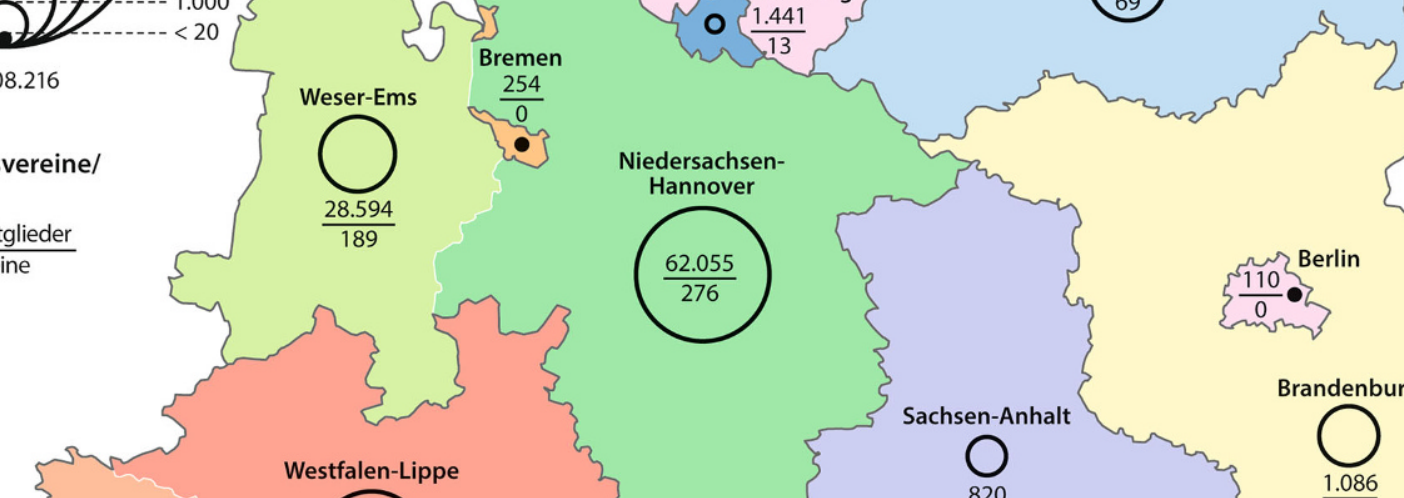

falen-Lipp
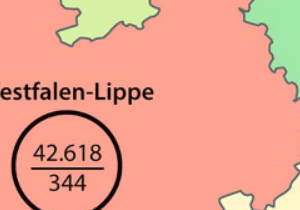

का
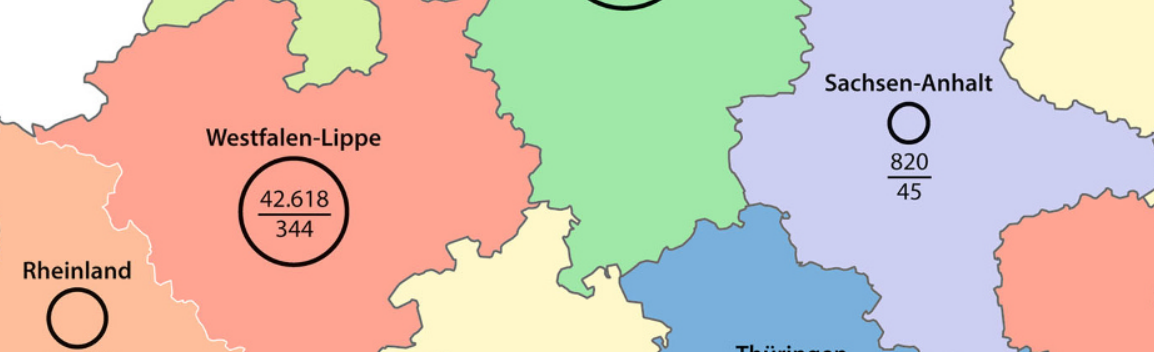

\section{Brandenburg}
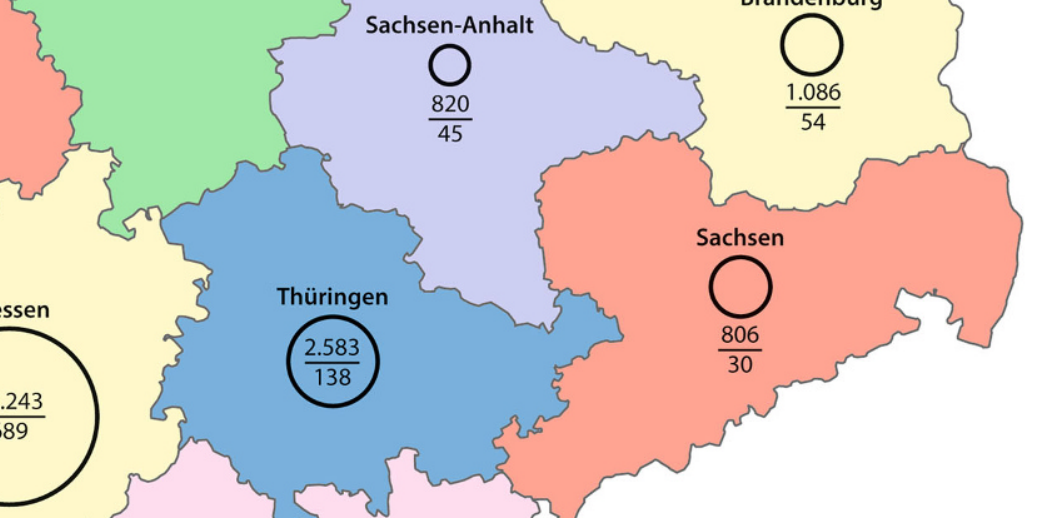

Rheinland-Nassau

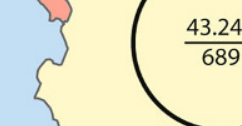

Abbildung 1 LandFrauenverbände in Deutschland Quelle: Eigene Darstellung auf der Basis von dlv (2016: 38 f.) 
Abbildung 2 Struktur und Organe der LandFrauenorganisationen Quelle: Eigene Darstellung auf der Basis von dlv (2016: $38 \mathrm{f}$.)

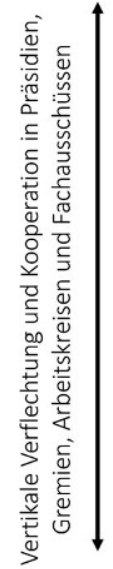

Bundesverband

Präsidium, Bundesvorstand,

Mitgliederversammlung, Fach-

ausschüsse, Bundesgeschäftsstelle

22 Landesverbände

Präsidium, Mitgliederversammlung, Arbeitskreise,

Landesgeschäftsstelle

422 Kreisverbände/Bezirksvereine

Vorstand und Geschäftsführung

10.679 Ortsvereine

Vorstand

450.000 Mitglieder

(inkl. Förder- und Ehrenmitglieder)

Horizontale Kooperationen zwischen Verbänden und Ortsvereinen

vom Land und der Europäischen Union gefördert werden (I 2; I 3; I 5).

Auf der Ebene der Landesverbände werden in Arbeitskreisen bzw. Fachausschüssen, die aus Delegierten der Kreisverbände bestehen, Themen gesetzt und diskutiert. Diese beraten den Vorstand inhaltlich und geben somit den Rahmen für die zukünftige Ausrichtung vor. Anregungen, Ideen und Bedarfe, die auf Orts- und Kreisvereinsebene entstehen bzw. wahrgenommen werden, werden somit in den Landesverband und darüber auch in den Bundesverband getragen. Der Vorstand bzw. das Präsidium repräsentiert den Verband nach außen, entscheidet über die Verbandsaktivität und setzt den Rahmen für zukünftige Veranstaltungen und Projekte (I 1; I 2; I 5).

Das Delegiertenprinzip und die inhaltliche Zusammenarbeit über Fachausschüsse bzw. Arbeitskreise setzen sich auf der Bundesebene fort. Den Bundesvorstand bilden Vorstände der Landesverbände, ,mit beratender Stimme die Geschäftsführerinnen der Landes-LandFrauenverbände sowie die Vorsitzende des Bundes der Deutschen Landjugend" (dlv o.J.a). Die Mitgliederversammlung setzt sich aus dem Bundesvorstand und den Delegierten der Landesverbände zusammen, wobei die Zahl der Delegierten im Verhältnis zur Mitgliederstärke der Landesverbände steht (dlv o.J.a).

Die Finanzierung der LandFrauenorganisationen erfolgt über die Vereinsmitgliedsbeiträge, wobei die Ortsvereine einen Teil an den Landesverband abführen, der wiederum einen Anteil an den Bundesverband weiterleitet (I 2). Die Ortsvereine nehmen teils zusätzlich über den Verkauf selbst gefertigter Produkte Geld ein (I 1; I 2; I 3; I 5). Über die Rückerstattung von Referentenkosten fließen Mittel von den Landesverbänden an die Ortsvereine zurück und die Landesverbände können Fördermittel über die Bundesebene für zentrale Informationsveranstaltungen, Arbeitskreise und Workshops im landwirtschaftlichen Bereich über den Bundesverband abrufen (I 2). Für Projekte werden Förder- mittel vom Bund und von Landesministerien sowie im Zuge der EU-Förderprogramme für den ländlichen Raum eingeworben. Des Weiteren werden Projekte oder Veranstaltungen über weitere Akteure, unter anderem Stiftungen, andere Vereine oder Verbände, Krankenkassen oder Unternehmen gefördert oder ko-finanziert (I 1; I 3; SVLFG 2013). Mittel erhalten der Bundesverband und die Vereine außerdem über das Instrument der Fördermitgliedschaft (I 1; I 2; I 3).

Mitgliederspektrum: Die soziale Struktur der LandFrauenorganisationen unterlag im zeitlichen Verlauf einem starken Wandel. Heute stehen die Ortsvereine Frauen aller Berufsgruppen offen, ${ }^{7}$ der Anteil an Bäuerinnen liegt heute bei ,ein[em] Drittel bis ein[em] Fünftel“ (Sawahn 2009: 13). Hinsichtlich der Altersstruktur bestehen große Unterschiede zwischen den Ortsvereinen: Wenngleich alle Altersstufen vertreten sind, ist ein Großteil der Mitglieder der Altersgruppe ab Mitte 50 zuzuordnen. Der demographische Wandel birgt damit Herausforderungen für die zukünftige Mitgliederentwicklung ${ }^{8}$ und auch für die Nachbesetzung ehrenamtlicher Funktionen (I 1; I 2; I 3). Diesem wird verbands- bzw. vereinsseitig begegnet durch neue Projekte, Angebote und Aktionen, die insbesondere Frauen jüngeren oder mittleren Alters ansprechen, sowie durch Qualifizierungs- und Unterstützungsangebote für ehrenamtliche Arbeit (I 1; I 2; I 5).

\subsection{Aktivität}

Als Ziele formuliert der Bundesverband „mehr Gerechtigkeit und Chancengleichheit für Frauen, die im ländlichen Raum zuhause sind. Dies beinhaltet die Wahrnehmung und Anerkennung der Leistungen und gleichberechtigte Teil-

\footnotetext{
${ }^{7}$ In Bayern ist die Mitgliedschaft an die berufliche Tätigkeit in einem Mitgliedsbetrieb des Bayerischen Bauernverbandes gebunden.

${ }^{8}$ Und steht damit in Bezug zu den eingehenden Mitgliedsbeiträgen.
} 
habe von LandFrauen in Beruf und Familie, in Gesellschaft und Politik, in Bildung, Wirtschaft und Kultur" (dlv o.J.a). Zur Umsetzung dieser Ziele werden auf allen geographischen Ebenen entsprechende Maßnahmen eingeleitet. Hervorzuheben sind erstens solche zur Vertretung der Interessen von Frauen und Landwirtinnen im ländlichen Raum, zweitens die Bildungs- und Qualifizierungsarbeit sowie drittens Projektarbeit. Die Aktivitätsspektren unterscheiden sich stark sowohl vertikal - von der Ebene des Ortsvereins bis zur Bundesebene - als auch horizontal zwischen Regionen und Vereinen (I 3; I 5). Im Folgenden wird auf diese Aktivität des Bundesverbands und der Landesverbände eingegangen, um anschließend lokal- und regionalwirksame Handlungen der Ortsvereine und Kreisverbände zu fokussieren.

Hauptaufgabe des Bundesverbandes ist die Vertretung und Bündelung der Interessen von Frauen im ländlichen Raum sowie die berufsständische Vertretung der Bäuerinnen (I 3; dlv o.J.a). Er bringt sich in politische Debatten ein, die die Interessen von Frauen, agrarwirtschaftliche Themen und Fragen der Entwicklung ländlicher Räume betreffen. Er bezieht Stellung über Positionspapiere und Pressemeldungen und ist regelmäßig auf Fach-, Vernetzungs- und Austauschveranstaltungen vertreten bzw. lädt zu diesen ein (I 3). Der Bundesverband ist breit vernetzt, in diversen Gremien vertreten und steht, ,im Dialog mit Vertreterinnen und Vertretern der Bundesregierung, des Parlaments, aller Parteien, berufsständischer Verbände und Institutionen sowie nationaler und internationaler Organisationen"9 (dlv o.J.a).

Des Weiteren unterstützt und bündelt der Bundesverband die Aktivität und Interessen der Landes- und Kreisverbände sowie der Ortsvereine über die dargestellte Organisationsstruktur aus Präsidien, Gremien und Arbeitsgemeinschaften bzw. Fachausschüssen. Er koordiniert Aktionstage, Informationsveranstaltungen und weitere gemeinsame Aktionen der Ortsvereine sowie der Kreis- und Landesverbände, die dazu dienen, auf Themen und Interessen der LandFrauen aufmerksam zu machen (I 1; I 2; I 3). 2015 fanden in diesem Rahmen über 250 Veranstaltungen mit etwa 123.400 Teilnehmerinnen statt (dlv 2015c).

Die Landesverbände setzen die Interessenvertretung unterhalb der Bundesebene fort und stehen auf der Landesebene in Dialog und Kooperation mit Politik, Verwaltung und weiteren Partnern. Sie sind ebenfalls breit vernetzt und in Gremien und Ausschüssen zu frauenpolitischen Sachgebieten, zur Erwachsenenbildung, zu agrarwirtschaftlichen Themen und zu Fragen des ländlichen Raums vertreten (I 2). Die Landesverbände unterstützen die Kreisverbände und Ortsvereine organisatorisch und tragen die auf lo-

\footnotetext{
${ }^{9}$ Unter anderem der Deutsche Bauernverband, die Deutsche Gesellschaft für Ernährung, der Deutsche Frauenrat, der Weltlandfrauenverband (ACWW) (dlv o.J.a.).
}

kaler Ebene wahrgenommenen Problemstellungen und Bedürfnisse über die Vorstände, die Delegiertenversammlungen und die Arbeitskreise und Fachausschüsse in den Bundesverband. Die Vorstände der Landesverbände setzen die Themen, zu welchen Projekte, Aktionen und Veranstaltungen durchgeführt werden, und die Anregungen für Aktionen und Veranstaltungen auf lokaler Ebene bieten. Unterhalb der Landesverbände setzen sich die Ortsvereine und Kreisverbände ${ }^{10}$ auf lokaler Ebene für die Interessen von Frauen im ländlichen Raum ein (vgl. Kapitel 4.4) (I 1; I 2; I 3 ; I 5).

„Auf allen Verbandsebenen werden zahlreiche Veranstaltungen zur Weiterbildung, z. B. berufliche Qualifikation, aktuelle Gesellschafts- und Sozialpolitik, durchgeführt“" (dlv o.J.a). Schwerpunkt der Bildungsarbeit des Bundesverbands ist die Qualifizierung im Bereich von Ehrenamt und Verbandsmanagement (dlv o.J.a). Dies schafft einen institutionell-organisatorischen Rahmen für lokales und kommunalpolitisches Engagement. Die Landesverbände erarbeiten eigene Bildungsprogramme und schaffen über Referentenund Themenlisten einen Rahmen für die Bildungsarbeit der Ortsvereine. Auf Landesebene sind die Bildungseinrichtungen der ländlichen Erwachsenenbildung wichtige Partner (I 1; I 2; I 3). Jeder Ortsverein stellt ein Bildungsprogramm, das auf die wahrgenommenen Interessen, Bedürfnisse und Begebenheiten vor Ort zugeschnitten ist. Die Angebote und Programme der Ortsvereine entspringen somit der Einbettung der Ortsvereine und der Vereinsmitglieder in lokale und soziale Kontexte und deren Wahrnehmung lokaler Begebenheiten, Interessen und infrastruktureller Bedarfe. Die Ortsvereine können auf das Referenten- und Bildungsangebot der Landesverbände bzw. des Bundesverbands zurückgreifen oder auch davon unabhängig eigene Referenten einladen. Die Veranstaltungen der Ortsvereine stehen zumeist auch Nicht-Mitgliedern offen und bieten damit auch einen Beitrag zur kulturellen Infrastruktur der Vereinsorte (I 1; I 2; I 3; I 4; I 5). Die Weiterbildungsstruktur basiert häufig auf dem Konzept, über Angebote auf Bundesebene Expertinnen auszubilden, die dann auf lokaler Ebene als Multiplikatorinnen ihr Wissen weitergeben (BMFSFJ 2004: 124).

Die Breite des Themenspektrums auf allen Ebenen zeigt Tabelle 1, die Art der Veranstaltungen nach Anzahl und Teilnehmerzahl Tabelle 2. Die Gesamtzahl an Veranstaltungen lag 2015 bei 108.216. ${ }^{11}$ Ein Schwerpunkt der Angebote spricht die persönlichen Lebens- und Interessenbereiche der Mitglieder an (vgl. Tabelle 1), gefolgt von umfeldbezoge-

\footnotetext{
${ }^{10}$ In einigen Bundesländern: Bezirksvereine oder -verbände.

11 Zum Vergleich: Die 905 Volkshochschulen in Deutschland boten 2015 insgesamt 591.603 Veranstaltungen mit 6.479.573 Belegungen durch Teilnehmer an, davon 195.954 zum Themenbereich Gesundheit und 90.972 zum Themenbereich „Kultur-Gestalten“ (Huntemann/ Reichart 2016: 29 ff.).
} 
Tabelle 1 Veranstaltungen nach Sachgebieten 2013-2015
Tabelle 2 Veranstaltungsformate 2015

\begin{tabular}{llll}
\hline Veranstaltungen nach Sachgebieten & $\begin{array}{l}\text { Anzahl } \\
\mathbf{2 0 1 5}^{\mathbf{a}}\end{array}$ & $\mathbf{2 0 1 4}$ & $\mathbf{2 0 1 3}$ \\
Gesundheit, Sport & 36.801 & 35.882 & 36.196 \\
Kunst, Kultur, Kreativität & 24.575 & 22.861 & 25.378 \\
Verbraucherpolitik, Hauswirtschaft, Ernäh- & 11.629 & 10.146 & 12.704 \\
rung & & & \\
Familie, Soziales & 6.819 & 6.922 & 7.490 \\
Beiträge für das Gemeinwohl (soziale Ar- & 5.386 & 6.014 & 6.468 \\
beiten) & & & \\
Landwirtschaft, ländlicher Raum, Umwelt & 5.343 & 5.239 & 5.662 \\
Kommunikation, Verbandsmanagement & 4.572 & 3.917 & 4.545 \\
Gesellschafts- und Sozialpolitik & 3.244 & 3.212 & 3.591 \\
Medienkompetenz, EDV & 892 & 1.208 & 1.051 \\
Wirtschaftspolitik & 748 & 805 & 1.046 \\
Sonstige & 8.207 & 9.204 & 8.832 \\
Insgesamt & $\mathbf{1 0 8 . 2 1 6}$ & $\mathbf{1 0 5 . 4 1 0}$ & $\mathbf{1 1 2 . 9 6 3}$ \\
\hline
\end{tabular}

a keine Angabe für den Landesverband Bremen sowie für das Sachgebiet „Beiträge für das Gemeinwohl“ in Bayern Quellen: dlv (2014: 38); dlv (2015b: 38); dlv (2016: 38)

\begin{tabular}{lll}
\hline Veranstaltungsformate $^{\mathbf{a}}$ & Anzahl & Teilnehmer $^{\mathbf{b}}$ \\
Kurs- und Seminartage & 42.700 & 669.291 \\
Vortrags- und Diskussionsveranstaltungen & 22.854 & 699.522 \\
Lehr- und Besichtigungsfahrten & 11.209 & 332.976 \\
Arbeitsgruppen & 9.825 & 109.493 \\
Ausstellungen und Märkte & 2.313 & k.A. \\
Sonstige Veranstaltungen & 18.345 & 462.046 \\
Insgesamt & $\mathbf{1 0 7 . 2 4 6}$ & $\mathbf{2 . 2 7 3 . 3 2 8}$ \\
\hline a nicht erfasst/gemeldet wurden Arbeitsgruppen, Ausstellungen/Märkte sowie sonstige Veranstaltungen der \\
LandFrauengruppe im Bayerischen Bauernverband \\
b Belegung je Veranstaltung \\
Quelle: dlv (2016: 39)
\end{tabular}

nen Aktivitäten. Darüber hinaus wird auch berufsbezogene Qualifizierung angeboten. So können sich Teilnehmerinnen etwa als Übungsleiterin für präventive Gymnastik, Agrarbotschafterin oder Kräuterführerin ausbilden lassen, um anschließend in diesem Bereich berufliche Tätigkeiten zu verfolgen (I 1; I 2; I 5). EDV-Fortbildungen haben sich in vielen Landesverbänden als fester Bestandteil des Bildungsangebotes der LandFrauenorganisationen etabliert (vgl. BMFSFJ 2004).

Ein weiterer Tätigkeitsschwerpunkt im Zuge der Verbandsziele ist die Durchführung von bzw. die Initiierung oder Beteiligung an nationalen und regionalen Projekten. Vielfach ist der Bundesverband dabei Träger oder Initiator von (Modell-)Projekten, die auf Landes-, Kreis- oder Ortsvereinsebene fort- oder umgesetzt werden. Daneben sind die Verbände bzw. Vereine selbst Träger und Initiatoren von Projekten oder Aktionen. Die Landesverbände arbeiten dabei „eng [...] mit regionalen Einrichtungen, Landesinstitutionen und Ministerien“ zusammen (Snelinski 2005: 17).
Auch die Projektarbeit verfolgt das Ziel, die Situation von Frauen im ländlichen Raum zu verbessern. Viele Projekte sollen Einkommens- und Erwerbsmöglichkeiten für Frauen schaffen - einerseits über Qualifizierungsmaßnahmen, andererseits über die Förderung weiblichen Unternehmertums und durch Vernetzungsangebote (I 2; I 3). Ein weiterer Themenkomplex der Projektarbeit ist die Entwicklung des ländlichen Raums. Eng mit der Interessenvertretung verzahnt sind etwa die Aktivitäten für Chancengerechtigkeit. In diesem Rahmen sind unter anderem regionale „EqualPay-Beraterinnen" aktiv, die als Multiplikatorinnen über die Folgen erwerbsbiographischer Entscheidungen informieren (dlv o.J.d). Weitere Projekte stehen in Bezug zu agrarwirtschaftlichen Themen, zu den Interessen oder Produkten landwirtschaftlicher Betriebe oder beziehen sich auf lokales Brauchtum (I 3).

Im Gesundheitsbereich sind der Bundesverband und die Landesverbände etwa mit Projekten und Kampagnen im Bereich der Sturzprävention und der Brustkrebsprävention 
aktiv (dlv o.J.f). Bewusstsein für ernährungs- und gesundheitspolitische Themen und Verbraucherbildung wird etwa im Zuge der bundesweiten Projekte „Schmexperten“ sowie „Gartenkinder“" geschaffen (I 3; dlv o.J.e). Im Themenbereich der Gesellschafts- und Sozialpolitik umfasst die Projektarbeit beispielsweise Aktivitäten zur Stärkung demokratischer Strukturen. Neben Qualifizierungen für das Ehrenamt führen die Landesverbände und der Bundesverband auch Fortbildungen und Projekte zur Förderung weiblichen Engagements in der Kommunalpolitik durch (I 2; I 3).

Eine inhaltliche Klammer der Projekte und die Förderung von Erfahrungs- und Ideenaustausch erfolgt durch Workshops und Arbeitsgemeinschaften der Landesverbände sowie durch gemeinsame Veranstaltungen zwischen Ortsvereinen oder Kreisverbänden (I 1; I 2). Über die Bundesebene hinaus werden auch internationale Projekte verfolgt. So unterstützt der Bundesverband etwa dauerhaft Projekte der Welthungerhilfe (I 3). ${ }^{12}$

\subsection{Lokales Engagement auf der Ebene der Ortsvereine und Kreisverbände}

Die lokale Dynamik der Ortsvereine und Kreisverbände variiert sehr stark; sie kann als Ausdruck des jeweiligen spezifischen dörflichen (oder städtischen) Kontextes verstanden werden. Das Veranstaltungsprogramm und das weitere umfeldbezogene Engagement entstehen in Reaktion auf wahrgenommene lokale Interessen, Bedarfe und infrastrukturelle Defizite und sind entsprechend auf diese zugeschnitten. In der Planung und Umsetzung ihrer Aktivitäten sind die Ortsvereine unabhängig, erfahren aber vielfältige Unterstützung und konzeptionelle Rahmung durch die Dachverbände (I 1; I 2; I 3; I 5).

Viele Aktivitäten bezüglich der Vertretung weiblicher Interessen finden auf Ortsebene statt, etwa Initiativen und Unterschriftenaktionen, die auf die Belange von Frauen aufmerksam machen. Auf lokaler Ebene sind LandFrauen unter anderem in Gemeindevertretungen, Beiräten, runden Tischen und Kreistagen vertreten (I 2; I 3; I 5). Die Ortsvereine sind auf lokaler Ebene wichtige Träger von Erwachsenenbildung (vgl. Kapitel 4.3), gleichzeitig leisten sie über ihre Veranstaltungsangebote Beiträge zur sozialen und kulturellen Infrastruktur. Auf der lokalen Ebene finden vielfältige soziale Aktionen statt, organisiert durch den Ortsoder Kreisvorstand, etwa gemeinsame Feiern, Ausflüge sowie Lehr- und Besichtigungsfahrten (I 1; I 2; I 3; I 4; I 5). Das soziale Miteinander, die Veranstaltungsangebote, die Erweiterung des eigenen Bekanntenkreises und Horizonts und auch die durch andere Mitglieder erfahrene Unterstützung wurden seitens aller Interviewpartnerinnen als

\footnotetext{
12 Derzeit das Projekt „LandFrauen für Uganda“. Für 2017 ist ein Pro-
} jekt in Ghana in Planung (I 3). wichtige Aspekte der Mitgliedschaft hervorgehoben. Die LandFrauenvereine bilden „einen bedeutenden verbindenden Faktor im kulturellen ländlichen Leben [und] ermöglichen alteingesessenen wie neu hinzuziehenden Frauen in den Dörfern in spezifischer Weise ein Gemeinschaftsleben, wie es keine andere Institution verlässlich bietet" (Sawahn 2009: 13).

Ebenso sind die Ortsvereine an lokalen und dörflichen Veranstaltungen anderer lokaler Gruppen beteiligt. Viele Aktivitäten erfolgen in Kooperation mit lokalen Vereinen und kommunalen Vertretern. ${ }^{13}$ Bei externen Veranstaltungen übernehmen LandFrauen häufig die kulinarische Versorgung (I 1; I 2; I 4). Das bürgerschaftliche Engagement der LandFrauen vor Ort erstreckt sich etwa auf Unterstützung zum Erhalt oder Aufbau lokaler Infrastruktur sowie auf den Kinder- und Jugendbereich. Verschiedene Ortsvereine greifen derzeit die Thematik der Migration und der Flüchtlinge auf (dlv 2015a: 6 ff.; I 3; I 5). Teils werden diese Tätigkeiten als längerfristige oder dauerhafte Projekte konzipiert. Weiterhin beziehen der Bundesverband wie auch verschiedene Landesverbände über Positionspapiere Stellung zur Thematik, indem sie z. B. auf die Chancen der Migration für ländliche Räume verweisen, Handlungsbedarfe und Forderungen an die Politik formulieren und ihre Ziele und Forderungen in andere Organisationen tragen. Orts-, Kreis- und Landesverbände führen darüber hinaus Spendenaktivitäten zugunsten regionaler Einrichtungen und Projekte durch (I 1; I 2; I 3).

LandFrauen sind als Partner an koordinierten regionalen Entwicklungsströmungen beteiligt, sind in lokalen Gremien, Arbeitskreisen und Ausschüssen aktiv und engagieren sich in lokalen Netzwerken, etwa im Zuge der Lokalen Agenda 21 und in lokalen LEADER-Aktionsgruppen. Diese Aktivitäten werden seitens der Landesverbände unter anderem im Rahmen von Arbeitskreisen aufgearbeitet, es werden Workshops zum Erfahrungsaustausch angeboten sowie Informationsveranstaltungen durchgeführt (I 2; I 3). Verschiedene Maßnahmen sowohl auf Orts- und Kreis- als auch auf Landes- und Bundesebene weisen Bezüge zur lokalen Wirtschaft auf und dienen der Förderung von Arbeitsplätzen und der Einkommenssicherung für Frauen im ländlichen Raum (Krieg 1999: 97). Neben der Interessenvertretung, den aufgeführten Bildungsangeboten und den Qualifizierungslehrgängen gilt dies für verschiedene Projekte, etwa zur Förderung von Unternehmerinnentum durch die Unterstützung von Existenzgründung. ${ }^{14}$ LandFrauen bieten, ein-

\footnotetext{
${ }^{13}$ Eine Intensivierung lokaler Vernetzung wird dabei mithilfe von Digitalisierung angestrebt (dlv 2017).

${ }^{14}$ In Ostdeutschland wurden etwa im Rahmen des Modellprojektes „SELF - Frauen gestalten Strukturentwicklungen ländlicher Regionen“ 25 Existenzgründungen und 40 Arbeitsplätze geschaffen (Putzing 2003: 58).
} 
Abbildung 3 Funktionen der LandFrauen im ländlichen Raum

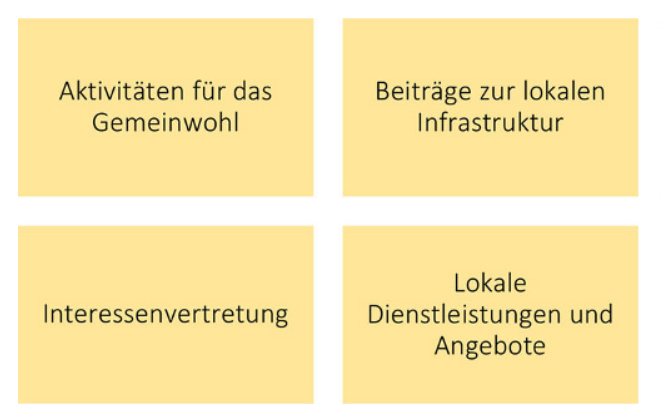

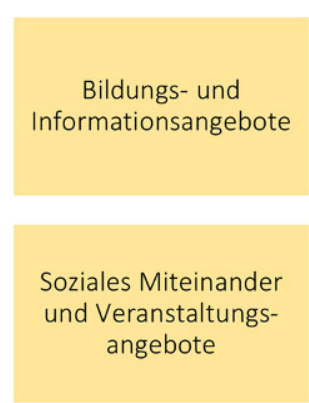

gebettet in die institutionell-organisationale Struktur ihrer Vereine und Verbände, vielfältige Dienstleistungen an. Im Haupt- oder Nebenerwerb vermarkten und verkaufen sie regionale Produkte, in den Bereichen Tourismus, Urlaub und Freizeit im ländlichen Raum sind die LandFrauen als lokale Gästeführerinnen aktiv bzw. initiieren oder tragen regionale Projekte (I 1; I 2). Daneben bestehen weitere Angebote in den Bereichen Ernährung, Gesundheit, Haushalt, Catering, Kinder und Altenbetreuung und -pflege. Viele davon sind im „LandPortal“ des Online-Auftritts des Bundesverbandes veröffentlicht (dlv o.J.b).

\section{Reflexion der regionalen und gesellschaftlichen Bedeutung der LandFrauenorganisationen}

Bürgerschaftliches Engagement vollzieht sich in weiten Teilen lokal oder regional (Schulte 2015: $31 \mathrm{ff}$.; Simonson/ Vogel/Tesch-Römer 2016: 538) innerhalb sozialer, ökonomischer und politischer Kontexte und Strukturen (Zimmer 2009; Nährlich 2011: 59). Gualini (2010: 15) diskutiert Potenziale von Zivilgesellschaft und bürgerschaftlichem Engagement für Stadtentwicklung und verweist auf einen ,inhärenten und konstitutiven" Raumbezug von Zivilgesellschaft und bürgerschaftlichem Engagement. Verschiedene Arbeiten diskutieren die Bedeutung bürgerschaftlichen Engagements für regionale oder lokale Entwicklung bzw. heben die Bedeutung von zivilgesellschaftlicher Infrastruktur und Vereinswesen hervor. So werden Zusammenhänge zu wirtschaftlicher Aktivität, dem Wohlstand oder der wirtschaftlichen Leistungsfähigkeit von Regionen konstatiert (Prognos 2009: 26; Franzen/Botzen 2011; Nährlich 2011: 55). Auch der „Freiwilligensurvey 2014“ identifizierte für Regionen mit niedriger Arbeitslosigkeit eine statistisch signifikant höhere Engagementquote (Simonson/Vogel/TeschRömer 2016: 552). Eine Vielzahl an Arbeiten betont die Bedeutung von Vereinen bzw. Zivilgesellschaft als Träger oder Generator von Sozialkapital (z. B. Braun 2005; Born 2014).

Das Aktivitätsspektrum der LandFrauenbewegung (vgl. Abbildung 3) weist eine einzigartige Kombination aus emanzipatorischen, sozialen und ökonomischen Interessen auf, die sich in den Vereins- und Verbandsaufgaben widerspiegelt. Im Hinblick auf ihr Engagement insbesondere in ländlichen Räumen ist die LandFrauenbewegung damit aus wirtschafts- und sozialgeographischer Perspektive hochrelevant, hat jedoch bislang wenig Aufmerksamkeit in der Forschung zu Zivilgesellschaft und Regionalentwicklung erfahren.

\subsection{Lokale Dienstleistungen, Daseinsvorsorge und lokale Infrastruktur}

Vielfach wird in ländlichen Regionen Deutschlands eine defizitäre infrastrukturelle Situation konstatiert. Das Ziel gleichwertiger Lebensverhältnisse in allen Teilräumen wird zunehmend im Zuge regional differenzierter Wachstumspolitik verfolgt und bedingt eine ,stärkere regionale Differenzierung der Lebensverhältnisse" (Barlösius 2009: 22 f.). Neu (2014: 118) verweist auf die Funktionen ,sozialer und territorialer gesellschaftliche[r] Integration " infrastruktureller Daseinsvorsorge. Damit ist insbesondere für schrumpfende und periphere Regionen die Bedeutung zivilgesellschaftlicher Akteure hervorzuheben: Bürgerschaftlich engagierte Akteure wirken als Bereitsteller von Daseinsvorsorge (Steinführer 2015), lokaler Dienstleistungen und Versorgung (Zimmer 2007b: 83; Evers 2010; Nährlich 2011: 57; Neu 2014).

Die vorherigen Ausführungen zeigen, dass die LandFrauenorganisationen in lokalen Kontexten zur (Weiter-)Bildungsinfrastruktur und zum Erhalt oder Aufbau sozialer wie auch kultureller Infrastruktur teils erheblich beitragen mit ihren Angeboten und Initiativen, die sich wohlgemerkt teilweise nur an die weibliche Bevölkerung und Mitglieder richten, jedoch auch vielfältige gesamtgesellschaftliche Fragen und vor Ort wahrgenommene soziale oder infrastrukturelle Probleme adressieren. Mit der Durchdringung des ländlichen Raums durch die Ortsvereine, deren Mitglieder in die Dorf- oder Quartiersgemeinschaften integriert sind, kommt den LandFrauen hohes Potenzial zu, lokale Bedürfnisse und Probleme zu erkennen und diese im Rahmen ihres organisationalen Kontextes anzugehen bzw. die Themen in die landes- und bundesweit agierenden Dachverbände zu kommunizieren und dort auf die Agenda 
der Interessenvertretungs-, Bildungs- und Projektaktivität zu setzen. Klie und Marzluff (2012: 749) identifizieren einen Rückgang von Versorgungsleistungen unter anderem in den Themenfeldern „Kulturelle Bildung“, „Pflege und Senioren“ sowie „Nahversorgung und Begegnung“ und verweisen auf die Potenziale der „Aktivierung verbleibender Bewohner der Dörfer“ zur „gemeinschaftlichen Sicherung der Versorgung“. Zu dieser tragen die LandFrauen im Zuge ihres Bildungs- und Veranstaltungsprogramms, mittels ihrer beruflichen Qualifizierung, durch ihre Beiträge zur lokalen sozialen Integration sowie in Hinblick auf ihre seniorenund pflegebezogenen Projekte und Dienstleistungen (vgl. Kapitel 4.3) umfangreich bei und entfalten somit neue „soziale Zugangs- und Teilhabemöglichkeiten“ (Neu 2009: 12).

\subsection{Ko-Produktion öffentlicher Güter und Akteure im Wohlfahrtspluralismus}

Zivilgesellschaftlichen Akteuren wird zunehmend Bedeutung als Ko-Produzenten öffentlicher Güter (Fürst 2012: 363) bzw. kollektiver Güter zugeschrieben und deren Rolle in diesbezüglichen Verteilungsfragen und Aushandlungsprozessen diskutiert (Hasenöhrl 2005). Aus der Perspektive der ökonomischen Gütertheorie lassen sich die Unternehmungen der LandFrauen bzw. deren Ergebnisse in öffentliche Güter, Clubgüter und private Güter kategorisieren. ${ }^{15}$ Öffentliche Güter zeichnen sich durch die Unmöglichkeit des Ausschlusses von Personen sowie durch Abwesenheit von Rivalität in der Nutzung aus (Ostrom 2005: 24). Hierunter lassen sich diejenigen Aktivitäten und Angebote der LandFrauen subsumieren, die das Gemeinwohl adressieren, mit gesellschaftlicher Wohlfahrt in Beziehung stehen bzw. gesamtgesellschaftliche Vorteile sowie Beiträge zur lokalen Infrastruktur schaffen. ${ }^{16}$ Je nach Grad des Lokalbezugs und geographischem Radius ist dabei von lokalen öffentlichen Gütern zu sprechen. Dies inkludiert auch Beiträge zum lokalen sozialen Kapital (Born 2014: 21). Ebenfalls können zivilgesellschaftliche Organisationen selbst mit ihren Angeboten der Partizipation und der Interessenartikulation als öffentliche Güter verstanden werden (Powell/Clemens 1998: xv). Hier ist unter anderem der Aufbau sozialer Infrastruktur durch die LandFrauen anzuführen. Als Clubgüter - für die Möglichkeiten des Ausschlusses vorliegen, jedoch keine Rivalität - sind jene LandFrauenaktivitäten und -angebote $\mathrm{zu}$ bezeichnen, die sich ausschließlich an die weibliche Bevölkerung bzw. an Mitglieder richten (vgl. Tabelle 2). Mit einer wachsenden Zahl an Mitgliedern und engagier-

\footnotetext{
15 Für detailliertere Ausdifferenzierungen der ökonomischen Gütertheorie vgl. Jansen und Priddat (2007).

${ }^{16}$ Etwa soziale und integrative Projekte, lokale Versorgungsangebote und Aktivitäten zum Erhalt lokaler Infrastruktur (vgl. Kapitel 4.3).
}

ten LandFrauen kann dabei ein wachsender Nutzen für die Mitglieder erwartet werden, etwa aufgrund breiterer Veranstaltungsangebote, zunehmender sozialer Kontakte und aufgrund einer höheren Wirksamkeit der Interessenvertretung. Dies entspricht dem Konzept der Netzwerkgüter als Sonderform der Clubgüter (vgl. Katz/Shapiro 1994; Glückler/ Hammer 2015). Daneben bestehen private Güter als individuelle Vorteile, die sich für die einzelnen Mitglieder oder die Adressaten der Maßnahmen ergeben, beispielsweise die Bereitstellung kulinarischer Angebote und die auf Basis der Qualifizierungsmaßnahmen angebotenen Dienstleistungen.

Im Hinblick auf ihre gesellschaftlichen Beiträge und auf öffentliche Güter können die LandFrauen als Element eines Wohlfahrtspluralismus verstanden werden, der auf gemeinsamer Wohlfahrtsproduktion von Akteuren aus Staat, Markt und Zivilgesellschaft basiert (Evers/Olk 1996: $8 \mathrm{ff}$.). Barlösius (2009: 25) verweist auf den Übergang vom vorsorgenden zum gewährleistenden Staat, verbunden mit einer „neuen Arbeitsteilung zwischen Staat, Markt und Zivilgesellschaft". Hierbei sei auf die Unterscheidung zwischen kompensatorischen, das heißt reduzierte staatliche Leistungen ausgleichende, und komplementären Funktionen von Zivilgesellschaft verwiesen (Braun 2004). Mit ihren Beiträgen zur lokalen Infrastruktur und der Übernahme von Aspekten der Daseinsvorsorge (vgl. Kapitel 4.3 und 5.1) ist den LandFrauen einerseits kompensatorische Funktion zuzuschreiben, gleichzeitig sind viele ihrer Engagements komplementär zu staatlichen Aufgaben und Angeboten, liefern die LandFrauenorganisationen doch Veranstaltungs-, Bildungs-, Partizipations- und Interessenartikulationsangebote, die andernfalls wohl ausbleiben würden.

\subsection{Die LandFrauen als lokale Partner und Akteure in Regional-Governance-Prozessen ${ }^{17}$}

„[Regional Governance] Arrangements ergänzen marktliche und staatliche Steuerungsmodi über Konzepte der kooperativen Erstellung von Gemeinschaftsgütern“ (Fürst 2012: 370). Dabei bildet die Partizipation zivilgesellschaftlicher Akteure ein wichtiges Element regionaler oder lokaler Governance (Walk/Dienel 2009: 197; Fürst 2012: $361 \mathrm{ff}$.). Walk (2014: 197f.) sieht Potenziale verstärkter bürgerschaftlicher Partizipation im Zuge lokaler Governance in Hinblick auf neue Lösungs- und Steuerungsformen, aber auch einhergehende Risiken der Entdemokratisierung sowie der Kompensierung reduzierter öffentlicher Leistungen. In der ländlichen Regionalentwicklung

\footnotetext{
17 Zum Konzept der Regional Governance vgl. Fürst (2012: 361): „Die freiwillige, aufgabenbezogene Kooperation von Akteuren aus dem öffentlichen, privatwirtschaftlichen und zivilgesellschaftlichen Bereich in netzwerkförmiger Organisationsstruktur über selbst gesetzte Regeln und Tauschhandel“".
} 
verweisen von Rohr und Wotha (2006: $11 \mathrm{f}$.) auf Funktionen Ehrenamtlicher als „Multiplikatoren“, die ,inhaltliche Diskussionen tragen“, „Ergebnisse verbreiten“ und „die Grundlage der Netzwerke und des kreativen Millieus im ländlichen Raum bilden", Projekte initiieren und umsetzen sowie fachliche Arbeit leisten.

In Forschung und Praxis wird vielfach die Bedeutung lokaler Netzwerke und sozialen Kapitals für Regionalentwicklungsmaßnahmen und regionale bzw. lokale Governance diskutiert (vgl. Fürst 2004; Scheer 2008). Die LandFrauen treten verschiedentlich als Partner von lokalen Bündnissen und koordinierten Regionalentwicklungsprojekten auf und setzen sich auf kommunaler Ebene für die Interessen von Frauen im ländlichen Raum ein. Sie bilden ein stabiles organisationales Netzwerk, das einerseits über die personelle Vernetzung seiner Mitglieder stark lokal verwurzelt ist. Das breite, berufsgruppen- und altersübergreifende Mitgliederspektrum und die Vielzahl lokaler Ortsvereine der LandFrauen sind in diesem Kontext hervorzuheben. Andererseits sind insbesondere die Landesverbände und der Bundesverband breit in überregionale Strukturen und Gremien eingebunden und mit politischen und wirtschaftlichen Akteuren vernetzt. ${ }^{18}$

Damit ist von sehr breiten und ausdifferenzierten Beitragsmöglichkeiten der LandFrauen im Zuge regionaler Governance auszugehen. So können LandFrauen auf lokaler Ebene vielfältig zwischen verschiedenen lokalen Gruppen vermitteln bzw. Kontakte aufbauen. Sie können eine breite Personengruppe vor Ort repräsentieren oder erreichen und diese zur Partizipation in lokalen Governance-Prozessen aktivieren. Krambach (2005: 7 f.) verweist auf die Potenziale der LandFrauen als ,gemeinschaftliche lokale Kraft" und lokale Mobilisatoren. Die Vermittlung zwischen ,unterschiedlichen Dorfkulturen" wurde durch die LandFrauen selbst explizit als Eigenanspruch formuliert (dlv 1998: 122). Gleichzeitig fördern sie lokale Beschäftigung durch ihre Qualifizierungsangebote und ihre Unterstützung von Unternehmerinnentum und lokalen Dienstleistungsangeboten (vgl. Kapitel 4.4). Koch (2012: 111) führt aus, dass zur Umsetzung nachhaltiger Dorfentwicklung in Zusammenarbeit mit der dörflichen Bevölkerung ,jedes Dorf individuell betrachtet werden muss“. Auch diesbezüglich kann den LandFrauen als Partner Potenzial zugeschrieben werden.

\section{Forschungspotenziale hinsichtlich Geographien bürgerschaftlichen Engagements}

Aufgrund ihres Engagements, ihres breiten Mitgliederspektrums und ihrer Mitgliederstärke sowie ihrer hohen Durch-

18 Dies kann auf die von Walk (2014) konstatierten Risiken von Entdemokratisierung auch kritisch gesehen werden. dringung insbesondere des ländlichen Raums ist den LandFrauen gesellschaftliche Relevanz und Bedeutung als Forschungsgegenstand zuzusprechen. Zunächst sei nochmals auf die generell unzureichende Erforschung der LandFrauenorganisationen als zivilgesellschaftliche Organisationen verwiesen (vgl. Kapitel 3.2). Im Hinblick auf Forschungspotenziale erscheint es somit fruchtbar, die Rolle der LandFrauen als Träger von Engagement und als Akteur in Entwicklungsprozessen im ländlichen Raum zu analysieren, um weiter zur Diskussion der Funktionen und der Einbindung zivilgesellschaftlicher Akteure in die Governance und Entwicklung im ländlichen Raum beizutragen. Eine vertiefte Betrachtung dieser Organisationen ist somit unter anderem hinsichtlich folgender Forschungsdesiderata von Interesse:

Erstens kann eine Betrachtung der LandFrauen zu einem vertieften Verständnis der organisatorischen Rahmung bürgerschaftlichen Engagements und der Funktionsweise zivilgesellschaftlicher Organisationen beitragen: Verschiedentlich wird eine Nachfrage an qualitativen und MixedMethod-Ansätzen zu Zivilgesellschaft und an Analysen auf Mikroebene (Klein/Schwalb 2014: 8) bzw. auf kommunaler Ebene (Alscher/Priller 2011: 721) konstatiert. Olk (2011: 716) formuliert Forschungsbedarf hinsichtlich ,qualitative[r] Studien zur organisatorischen Einbettung des Engagements in Vereinen, Initiativen und Verbänden“, „um hemmende bzw. begünstigende Bedingungen für den Einbezug Engagierter in organisationale Strukturen und Handlungsabläufe identifizieren zu können“. Von besonderem Interesse kann dabei die Frage sein, inwieweit eine homogenisierende Wirkung durch die Dachverbände besteht und wie diese mit der organisationsspezifischen lokalen Einbettung zusammenwirkt.

Zweitens könnten in diesem Zuge weitere Erkenntnisse über die regionalen Disparitäten bürgerschaftlichen Engagements in Deutschland gewonnen werden. Verschiedene nationale Studien verweisen auf die lokalen Spezifika und geographischen Unterschiede bürgerschaftlichen Engagements (Prognos 2009; Krimmer/Priemer 2013; Generali Zukunftsfonds 2015; Simonson/Vogel/Tesch-Römer 2016). In ländlichen Regionen engagiert sich mit einer Engagementquote ${ }^{19}$ von 45,5 \% ein höherer Personenanteil als in Städten $(42,7 \%)$. Hierbei ist der Unterschied bei Männern jedoch deutlich stärker ausgeprägt als bei Frauen (Simonson/Vogel/Tesch-Römer 2016: 546 f.). ${ }^{20}$ Des Weiteren ließ sich für kleinere Kommunen eine höhere Engagementquote messen, was unter anderem auf gerin-

\footnotetext{
${ }_{19}$ Prozent der Wohnbevölkerung Deutschlands im Alter ab 14 Jahren (Simonson/Vogel/Tesch-Römer 2016: 85).

${ }^{20}$ Ausdifferenziert nach Siedlungsformen sind Frauen dagegen in städtischen Kreisen am stärksten engagiert (Simonson/Vogel/TeschRömer 2016: 550 f.).
} 
gere Bevölkerungsdichten und ,überschaubare Sozialbeziehungen" sowie die „urbane Infrastruktur" in Agglomerationsräumen zurückgeführt wird (Prognos 2009: 25). Verschiedene Autoren konstatieren Forschungsdefizite hinsichtlich Ausformungen bürgerschaftlichen Engagements in den neuen Bundesländern und verweisen auf diesbezügliche Unterschiede zu westdeutschen Kontexten. Olk (2011: 716) nennt Bedarfe an „Ost-West-Vergleichen“, um entsprechende Förder- bzw. Unterstützungsmodelle erarbeiten zu können. Alscher und Priller (2011: 721) vermissen organisationsbezogene Daten, ,insbesondere für die kommunalen Kontexte“. Die LandFrauenorganisationen sind in Ost- wie Westdeutschland, in ländlichen Räumen wie auch in Städten breit vertreten, Träger vielfältiger Formen bürgerschaftlichen Engagements und eingebunden in die regionale Entwicklungsarbeit. Eine regional ausdifferenzierte Betrachtung und Analyse ihrer Aktivitäten, ihrer Einbettung in lokale Kontexte in Wechselwirkung mit ihrer Organisation kann einen wesentlichen Beitrag zum Verständnis von organisational gerahmtem Engagement und dem Beitrag zivilgesellschaftlicher Akteure zu kommunaler Infrastruktur liefern. Insbesondere kann es sich lohnen, Spezifika der LandFrauenbewegung in den neuen Bundesländern stärker in den Blick zu nehmen.

Drittens kann eine Betrachtung der LandFrauen zur Klärung der Beziehung zwischen Zivilgesellschaft und öffentlichen Gütern beitragen (vgl. Klein 2014: 7). In Kapitel 5.1 wurden die LandFrauenaktivitäten aus der ökonomischen Gütertheorie heraus betrachtet. Mit der Transformation zum gewährleistenden oder aktivierenden Staat und mit zunehmender Aufgabenteilung wächst die Bedeutung von $\mathrm{Zi}$ vilgesellschaft für gesellschaftliche Wohlfahrtsproduktion und hinsichtlich der Produktion öffentlicher Güter (Jansen/Priddat 2007: 35; Barlösius 2009: 25). Wissenschaftliche Debatten umfassen unter anderem organisatorische und Governance-Fragen sowie damit einhergehende legitimatorische, politische und normative Probleme. Für kollektive Güter und Allmendegüter wird die Rolle zivilgesellschaftlicher Akteure in Aushandlungsprozessen bezüglich Nutzungs- und Verteilungsfragen betrachtet. Im Zuge einer Untersuchung der LandFrauenorganisationen lassen sich dagegen regional vergleichend die konkreten Prozesse, Ursachen und Rahmenbedingungen der Bereitstellung öffentlicher Güter durch diese zivilgesellschaftlichen Organisationen nachzeichnen.

Viertens ergibt sich die Gelegenheit, die Partizipation zivilgesellschaftlicher Akteure in lokaler Governance regional vergleichend zu analysieren und mit den Untersuchungen zu Geographien des Engagements in Beziehung zu setzen. ${ }^{21}$ Die lokalspezifischen Ortsvereine und Kreisverbände, die gleichzeitig durch die Einbindung in die Struktur der Landesverbände und des Bundesverbands einheitlichen Rahmenbedingungen unterliegen, bilden dafür interessante Ansatzpunkte. Mit ihrem lokalen Engagement reihen sich die LandFrauen ein in eine Vielfalt lokaler zivilgesellschaftlicher Akteure, mit denen sie in verschiedenartigen Interaktionen und Kooperationen stehen (vgl. Kapitel 4.3), welche ebenfalls einen relevanten Gegenstand einer vergleichenden Betrachtung lokaler Governancestrukturen darstellen. Weiterhin können die Leistungen, Erfahrungen und Entwicklungslinien des lokalen Engagements der LandFrauen wertvolle Anknüpfungspunkte für weiterführende Debatten hinsichtlich Problemen und Lösungen für ländliche Räume sein.

Fünftens können die LandFrauen in Hinblick auf feministische und Gender- bzw. geschlechterbezogene Zivilgesellschafts- sowie Lobby- und Verbändeforschung von Interesse sein (vgl. Backes 2011; Wilde 2014). Diesbezüglich könnten die LandFrauen als sehr spezifischer, auf weibliche Interessen fokussierter Interessenverband ein erkenntnisreicher Forschungsgegenstand sein.

\section{Ausblick und Forschungsagenda}

Dieser Beitrag formuliert die Grundlagen für eine weiterführende, regional vergleichende Betrachtung der heutigen LandFrauenorganisationen aus regionalentwicklungsbezogener Perspektive. Die Ausführungen verdeutlichen die gesellschaftliche Relevanz der LandFrauenorganisationen als ein wichtiges Element ländlicher Zivilgesellschaft sowie die wissenschaftlichen Potenziale ihrer Analyse für die sozialund wirtschaftsgeographische Forschung zu bürgerschaftlichem Engagement. Ebensowenig wie den ländlichen Raum gibt es den typischen LandFrauenverein/-verband - dieser entspringt jeweils seinem lokalen, sozialen und ökonomischen Umfeld. Insbesondere die Ortsvereine ergeben sich aus der lokalen Gemeinschaft und der sozialräumlichen Umgebung und ihre Aktivitäten können als Reaktion auf wahrgenommene lokale Bedürfnisse, Interessen und infrastrukturelle Probleme aufgefasst werden. Damit leisten die LandFrauen einen oft substanziellen und vor Ort sehr individuellen Beitrag für das Gemeinwesen und das dörfliche (oder städtische) Leben, der sich aus der Integration in eine strukturierende und rahmensetzende Verbandsstruktur in Verbindung mit der Individualität und organisationalen Eigenständigkeit der Ortsvereine ergibt.

\footnotetext{
${ }^{21}$ Die Notwendigkeit vergleichender Empirie sowie theoretischer Fundierung über die Einbindung zivilgesellschaftlicher Akteure in lokale Governance stellen etwa Geißel (2007: 36) und Zimmer (2007c: 106) fest.
} 
Im Zuge einer weiterführender Forschungsagenda wird das Ziel verfolgt, über die Betrachtung der LandFrauenbewegung mittels einer Kombination qualitativer und quantitativer Methoden zum Abbau der oben dargelegten Forschungsdefizite beizutragen, indem die spezifischen lokalen sozioökonomischen Einbettungen der LandFrauen in Wechselwirkung mit deren Beiträgen zur lokalen Infrastruktur und Daseinsvorsorge erhoben werden. Regional vergleichend soll dabei analysiert werden, wie die Bereitstellung von öffentlichen Gütern durch die LandFrauen als zivilgesellschaftliche Akteure organisiert und gerahmt wird, wie sich lokale Anpassungsmechanismen vollziehen und welche Bedeutung der sozioökonomische Kontext für regionale Disparitäten von Engagement hat. Auf der Basis einer solchen weiterführenden organisationsbezogenen Betrachtung könnten Rückschlüsse auf die lokale Kontextualisierung von Zivilgesellschaft gezogen werden. Gleichzeitig würde damit eine regional ausdifferenzierte Betrachtung der LandFrauen als zivilgesellschaftliche Organisationen geleistet, die mit dem bisherigen Stand der Forschung zu regionalen Disparitäten bürgerschaftlichen Engagements und zur Partizipation von Zivilgesellschaft in lokaler und regionaler Governance erweiternd in Beziehung gesetzt werden kann.

Danksagung Mein herzlicher Dank geht an die Interviewpartnerinnen und Kontaktpersonen der LandFrauen für ihre Zeit und ihre Unterstützung! Auch möchte ich mich herzlich bei den anonymen Reviewern und den Herausgebern für ihre hilfreichen Anmerkungen und konstruktive Kritik bedanken.

\section{Literatur}

Alscher, M.; Priller, E. (2011): Organisationsbezogene Daten. In: Olk, T.; Hartnuß, B. (Hrsg.): Handbuch Bürgerschaftliches Engagement. Weinheim/Basel, 719-731.

Ambos, I.; Greubel, S. (2012): Ökonomische Grundbildung für Erwachsene. Themenfeld „Akteurs- und Angebotsanalyse“. Bonn. http://www.die-bonn.de/doks/2012-oekonomische-grundbildungakteurs-und-angebotsanalyse-01.pdf (19.05.2017).

Backes, G. M. (2011): Geschlechterdifferenz im Engagement. In: Olk, T.; Hartnuß, B. (Hrsg.): Handbuch Bürgerschaftliches Engagement. Weinheim/Basel, 65-76.

Barlösius, E. (2009): Der Anteil des Räumlichen an sozialer Ungleichheit und sozialer Integration: Infrastrukturen und Daseinsvorsorge. In: Sozialer Fortschritt 58, 2/3, 22-28.

Baumgart, E.; Becker, U.; Borsch, K.; Merrem, J. (2004): Professionalisierung im Ehrenamt und zielgruppenorientierte Weiterbildung. Das Projekt „Lebenslanges Lernen“ im Landfrauenverband Bernkastel-Wittlich. In: Behrmann, D.; Schwarz, B.; Götz, K. (Hrsg.): Professionalisierung und Organisationsentwicklung: Optimierung der Rahmenbedingungen des lebenslangen Lernens in der Weiterbildung. Bielefeld, 249-258.

Biegler, D. (2001): Frauenverbände in Deutschland. Entwicklung, Strukturen, politische Einbindung. Opladen. $=$ Forschung Politikwissenschaft 139.

BMFSFJ - Bundesministerium für Familie, Senioren, Frauen und Jugend (2004): Neue Medien für LandFrauen - IT-Landfrauen. Verlauf und Ergebnisse nach drei Jahren Laufzeit des Modellprojekts. https://www.bmfsfj.de/blob/84326/b4df0409f128c817ced490bc02 ead584/langfassung-it-landfrauen-data.pdf (19.05.2017).
Born, D. (2014): Vereine als öffentliches Gut: Die sozio-politischen Wirkungen lokaler Schweizer Vereinslandschaften. Wiesbaden.

Braun, S. (2004): Die Wiederentdeckung des Vereinswesens im Windschatten gesellschaftlicher Krisen. In: Forschungsjournal Soziale Bewegungen 17, 1, 26-35.

Braun, S. (2005): Bürgergesellschaft und sozialer Zusammenhalt. Theoretische Überlegungen und empirische Befunde zu den Integrationsleistungen von freiwilligen Vereinigungen im Dritten Sektor. In: Birkhölzer, K.; Klein, A.; Priller, E.; Zimmer, A. (Hrsg.): Dritter Sektor, drittes System. Theorie, Funktionswandel und zivilgesellschaftliche Perspektiven. Wiesbaden, 131-159. = Bürgergesellschaft und Demokratie 20.

Bridenthal, R. (1994): Die Rolle der organisierten Landfrauen bei der konservativen Mobilmachung in der Weimarer Republik. In: Feministische Studien 12, 1, 110-121.

Bundesamt für Justiz (2016): Zusammenstellung der Geschäftsübersichten der Amtsgerichte für die Jahre 1995 bis 2015. https://www.bundesjustizamt.de/DE/SharedDocs/Publikationen/ Justizstatistik/Geschaeftsentwicklung_Amtsgerichte.pdf (19.05. 2017).

Deutscher Bundestag (2002): Bürgerschaftliches Engagement: Auf dem Weg in eine zukunftsfähige Bürgergesellschaft. Bericht der Enquete-Kommission „Zukunft des bürgerschaftlichen Engagements“. http://dip21.bundestag.de/dip21/btd/14/089/1408900.pdf (05.05.2017).

dlv - Deutscher Landfrauenverband (1998): Engagiert auf dem Land. Münster.

dlv - Deutscher LandFrauenverband (2014): LandFrauen mischen sich ein. Jahresbericht 2013. Berlin. http://www.landfrauen. info/fileadmin/Redaktion/PDF/Publikationen/dlv_Jahresbericht_ 2013.pdf (19.05.2017).

dlv - Deutscher LandFrauenverband (2015a): Willkommen heißen. In: LandFrauen aktuell 4, 6-9. http://www.landfrauen.info/fileadmin/ Redaktion/PDF/Publikationen/LF_aktuell/2015_LF_aktuell_4. pdf (19.05.2017).

dlv - Deutscher LandFrauenverband (2015b): LandFrauen mit Ideen. Jahresbericht 2014. Berlin. http://www.landfrauen.info/fileadmin/ Redaktion/PDF/Publikationen/dlv_Jahresbericht_2014.pdf (19. 05.2017).

dlv - Deutscher LandFrauenverband (2015c): Rückblick auf die Aktionstage. 2015 erfolgreich eingemischt. Pressemeldung vom 29. September 2015. http://www.landfrauen.info/fileadmin/Redaktion/ PDF/Mitmachen/2016_dlv_Rueckblick_Aktionstage_2015-2011. pdf (19.05.2017).

dlv - Deutscher LandFrauenverband (2016): LandFrauen mischen sich ein. Jahresbericht 2015. Berlin. http://www.landfrauen. info/fileadmin/Redaktion/PDF/Publikationen/dlv_Jahresbericht_ 2015_og.pdf (05.05.2017).

dlv - Deutscher LandFrauenverband (2017): Ehrenamt 4.0 - Chancen der Digitalisierung nutzen und Herausforderungen gemeinsam angehen. Pressemeldung vom 27. Januar 2017. http://www. landfrauen.info/aktuelles/pressemeldungen/meldung/ehrenamt40-chancen-der-digitalisierung-nutzen-und-herausforderungengemeinsam-angehen (19.05.2017).

dlv - Deutscher LandFrauenverband (o.J.a): Aus Liebe zum Land. Wofür aktive LandFrauen stehen. Ziele und Botschaften. http://www. landfrauen.info/verband/zielebotschaften/ (19.05.2017).

dlv - Deutscher LandFrauenverband (o.J.b): Das Landportal. Angebote für Neugierige. http://www.landfrauen.info/landportal/daslandportal/ (19.05.2017).

dlv - Deutscher LandFrauenverband (o.J.c): Die Geschichte des Deutschen LandFrauenverbandes. Von den Gründerjahren bis heute. http://www.landfrauen.info/verband/geschichte.html (19.05.2017).

dlv - Deutscher LandFrauenverband (o.J.d): Gerechte Chancen. EqualPay-Beraterinnen klären auf. http://www.landfrauen.info/themen/ gerechte-chancen/artikel/equal-pay-beraterinnen-klaeren-auf/ (19.05.2017). 
dlv - Deutscher LandFrauenverband (o.J.e): Kompetent im Alltag. http://www.landfrauen.info/themen/kompetent-im-alltag/ (19.05.2017).

dlv - Deutscher LandFrauenverband (o.J.f): Trittsicher durchs Leben. Sturzprävention. http://www.landfrauen.info/themen/kompetentim-alltag/artikel/trittsicher-durchs-lebensturzpraevention/ (19.05. 2017).

Evers, A. (2010): Zivilgesellschaft, Engagement und soziale Dienste. In: Olk, T.; Klein, A.; Hartnuß, B. (Hrsg.): Engagementpolitik. Die Entwicklung der Zivilgesellschaft als politische Aufgabe. Wiesbaden, 282-302.

Evers, A.; Olk, T. (1996): Wohlfahrtspluralismus - Analytische und normativ-politische Dimensionen eines Leitbegriffs. In: Evers, A.; Olk, T. (Hrsg.): Wohlfahrtspluralismus. Vom Wohlfahrtsstaat zur Wohlfahrtsgesellschaft. Opladen, 9-60.

Franzen, A.; Botzen, K. (2011): Vereine in Deutschland und ihr Beitrag zum Wohlstand der Regionen. In: Soziale Welt 62, 4, 391-413.

Fürst, D. (2004): Regional Governance. In: Benz, A. (Hrsg.): Governance. Regieren in komplexen Regelsystemen. Wiesbaden, 45-64.

Fürst, D. (2012): Regional Governance (RG) - was hat die deutsche Diskussion gebracht? In: Egner, B.; Haus, M.; Terizakis, G. (Hrsg.): Regieren. Festschrift für Hubert Heinelt. Wiesbaden, 361-377.

Geißel, B. (2007): Zur (Un-) Möglichkeit von Local Governance mit Zivilgesellschaft: Konzepte und empirische Befunde. In: Schwalb, L.; Walk, H. (Hrsg.): Local Governance. Mehr Transparenz und Bürgernähe? Wiesbaden, 23-38.

Generali Zukunftsfonds (2015): Generali Engagementatlas 2015. Rolle und Perspektiven Engagement unterstützender Einrichtungen in Deutschland. Köln. https://zukunftsfonds.generali-deutschland. de/wissen/2015-engagementatlas/ (19.05.2017).

Glückler, J.; Hammer, I. (2015): Kooperationsgewinne durch Netzwerkgüter. In: Kölner Schrift zum Wirtschaftsrecht 6, 1, 7-14.

Gualini, E. (2010): Zivilgesellschaftliches Handeln und bürgerschaftliches Engagement aus stadtentwicklungspolitischer Perspektive. Kritische Überlegungen zur Thematik. In: Becker, E.; Gualini, E.; Runkel, C.; Graf Strachwitz, R. (Hrsg.): Stadtentwicklung, Zivilgesellschaft und bürgerschaftliches Engagement. Stuttgart, 3-24.

Habermann, D. (1995): Beratung zur Schaffung von Arbeitsplätzen für Landfrauen in den neuen Bundesländern. Am Beispiel von Modellprojekten zur Dorfentwicklung „Einkommenssicherung durch Dorftourismus“. In: Henkel, G. (Hrsg.): Außerlandwirtschaftliche Arbeitsplätze im ländlichen Raum. Essen, 55-73. = Essener Geographische Arbeiten 26.

Hasenöhrl, U. (2005): Zivilgesellschaft, Gemeinwohl und Kollektivgüter. Berlin. = WZB-Discussion Paper SP IV 2005-401.

Helmle, S. (2009): „Wie man sich durchbeißen kann“. Landfrauenarbeit in Bayern nach 1945. In: Oedl-Wieser, T.; Darnhofer, I. (Hrsg.): Gender Issues in der Landwirtschaft. Wien, 2742. = Jahrbuch der Österreichischen Gesellschaft für Agrarökonomie $18(2)$.

Huntemann, H.; Reichart, E. (2016): Volkshochschul-Statistik: 52. Folge, Arbeitsjahr 2013. http://www.die-bonn.de/doks/2014volkshochschule-statistik-01.pdf (19.05.2017).

Icken, A. (2002): Der deutsche Frauenrat. Etablierte Frauenverbandsarbeit im gesellschaftlichen Wandel. Opladen.

Jansen, S. A.; Priddat, B. P. (2007): Theorien der Öffentlichen Güter: Rekonstruktionen sozialer Konstruktionen. Politik-und wirtschaftswissenschaftliche Korrekturvorschläge. In: Jansen, S. A.; Priddat, B. P.; Stehr, N. (Hrsg.): Die Zukunft des Öffentlichen. Wiesbaden, 11-48.

Kaschuba, G. (1996): Bildungsprozesse in den Biographien von Frauen in ländlichen Regionen. In: Report. Literatur- und Forschungsreport Weiterbildung 37, 53-66.

Katz, M. L.; Shapiro, C. (1994): Systems Competition and Network Effects. In: The Journal of Economic Perspectives 8, 2, 93-115.

Kistemann, E. (2003): Landfrauen in Nordrhein-Westfalen als Gästeführerinnen. Kulturlandschaftliche Aspekte in der Ausbildung. In:
Kulturlandschaft - Zeitschrift für angewandte historische Geographie 13, 1/2, 29-32.

Klein, A. (2014): Bürgerschaftliches Engagement im ländlichen Raum. Der strukturschwache ländliche Raum als Herausforderung neuer Kooperationskulturen. Rede im Zuge des Zukunftsforums Ländliche Entwicklung. http://www.zukunftsforum-laendlicheentwicklung.de/fileadmin/SITE_MASTER/content/Dokumente/ Downloads2014/Beitraege2014/AbschlKlein.pdf (19.05.2017).

Klein, A.; Schwalb, L. (2014): Engagementforschung als Teil der Zivilgesellschaftsforschung. Eine Forschungsagenda im Ausgang vom Engagementbericht der Bundesregierung. http://www. b-b-e.de/fileadmin/inhalte/PDF/publikationen/jb02_expertise_ \%20engagementforschung.pdf (05.05.2017).

Klie, T.; Marzluff, S. (2012): Engagement gestaltet ländliche Räume. Chancen und Grenzen bürgerschaftlichen Engagements zur kommunalen Daseinsvorsorge. In: Zeitschrift für Gerontologie und Geriatrie 45, 8, 748-755.

Koch, S. (2012): Nachhaltige Dorfentwicklung. Zukunft - Identität Tradition in nordhessischen Dörfern. Kassel.

Kocka, J. (2003): Zivilgesellschaft in historischer Perspektive. In: Forschungsjournal Neue Soziale Bewegungen 16, 2, 29-37.

Köhler, M.; Gonon, P. (2004): Maximen als „sanftes“ Instrument zur Qualitätssicherung in der ehrenamtlich organisierten Erwachsenenbildung. In: Report. Zeitschrift für Weiterbildungsforschung 27, 1, 196-202.

Krambach, K. (2005): Vorwort. In: Rosa-Luxemburg-Stiftung (Hrsg.): Landfrauen - treibende Kraft der Dorfentwicklung. Dokumentation einer Tagung des Gesprächskreises Ländlicher Raum am 3. Juni 2005 in Berlin. Berlin, 4-8.

Krieg, B. (1999): Landfrauenbewegung im Wandel. Ziele, Inhalte, Herausforderungen und Perspektiven. In: Heidrich, H. (Hrsg.): Frauenwelten. Arbeit, Leben, Politik und Perspektiven auf dem Land. Bad Windsheim, 79-98.

Krimmer, H.; Priemer, J. (2013): ZiviZ-Survey 2012. Zivilgesellschaft verstehen. http://www.ziviz.info/file/206/download?token=FZB9 CeV- (24.05.2017).

Lütt, S. (2007): Wiederansiedlung von 60 Wildpflanzenarten in Schleswig-Holstein. Das Jubiläumsprojekt des LandFrauenVerbandes. In: Kieler Notizen zur Pflanzenkunde 35, 60-71.

Mayring, P. (2015): Qualitative Inhaltsanalyse. Grundlagen und Techniken. Weinheim/Basel.

Moss, T.; Gudermann, R. (2009): Gemeinschaftsgüter im Dienst der Regionalentwicklung. Eine Einführung. In: Bernhardt, C.; Kilper, H.; Moss, T. (Hrsg.): Im Interesse des Gemeinwohls: Regionale Gemeinschaftsgüter in Geschichte, Politik und Planung. Frankfurt am Main, 11-27.

Nährlich, S. (2011): Spenden und Ehrenamt, Vereine und Stiftungen. Wozu braucht man das? In: Hurrelmann, K.; Picot, S.; Nährlich, S. (Hrsg.): Diskurs Service Learning. Unterricht und Bürgerengagement verbinden. Berlin, 55-63.

Naumann, M.; Reichert-Schick, A. (2015): Ländliche Infrastrukturen - Risiken, Anpassungserfordernisse und Handlungsoptionen. In: Raumforschung und Raumordnung 73, 1, 1-3.

Neu, C. (2009): Daseinsvorsorge. Eine Einführung. In: Neu, C. (Hrsg.): Daseinsvorsorge. Eine gesellschaftswissenschaftliche Annäherung. Wiesbaden, 9-19.

Neu, C. (2014): Ländliche Räume und Daseinsvorsorge. Bürgerschaftliches Engagement und Selbstaktivierung. In: Dünkel, F.; Herbst, M.; Schlegel, T. (Hrsg.): Think Rural! Dynamiken des Wandels in peripheren ländlichen Räumen und ihre Implikationen für die Daseinsvorsorge. Wiesbaden, 117-124.

Olk, T. (2011): Qualitative Forschung. In: Olk, T.; Hartnuß, B. (Hrsg.): Handbuch Bürgerschaftliches Engagement. Weinheim/Basel, 705-718.

Ostrom, E. (2005): Understanding Institutional Diversity. Princeton.

Powell, W. W.; Clemens, E. S. (1998): Introduction. In: Powell, W. W.; Clemens, E. S. (Hrsg.): Private Action and the Public Good. New Haven, xiii-xix. 
Prognos AG (2009): Engagementatlas 2009. Daten. Hintergründe. Volkswirtschaftlicher Nutzen. Aachen.

Putzing, M. (2003): Neue Erwerbsperspektiven für Frauen auf dem Lande. Ergebnisse eines innovativen Modellprojektes. In: Agrarbündnis (Hrsg.): Der Kritische Agrarbericht 2003. Rheda-Wiedenbrück, 58-62.

Putzing, M. (2005): Frauen bewegen das Land. Möglichkeiten und Grenzen der Beteiligung von Frauen an der Dorf- und Regionalentwicklung in den Neuen Bundesländern. In: Rosa-LuxemburgStiftung (Hrsg.): Landfrauen - treibende Kraft der Dorfentwicklung. Dokumentation einer Tagung des Gesprächskreises Ländlicher Raum am 3. Juni 2005 in Berlin. Berlin, 9-15.

Rosa-Luxemburg-Stiftung (Hrsg.) (2005): Landfrauen - treibende Kraft der Dorfentwicklung. Dokumentation einer Tagung des Gesprächskreises Ländlicher Raum am 3. Juni 2005 in Berlin. Berlin.

Rudolph, C.; Schirmer, U. (2004): Gestalten oder verwalten? Kommunale Frauenpolitik zwischen Verrechtlichung, Modernisierung und Frauenbewegung. Wiesbaden. = Politik und Geschlecht 14.

Sawahn, A. (2009): Die Frauenlobby vom Land. Die Landfrauenbewegung in Deutschland und ihre Funktionärinnen 1898 bis 1948. Frankfurt am Main.

Sawahn, A. (2012): „Tradition mit frischem Wind“. Weibliches Selbstbildnis als Machtfaktor der Landfrauenvereine. In: Münkel, D.; Uekötter, F. (Hrsg.): Das Bild des Bauern. Selbst- und Fremdwahrnehmungen vom Mittelalter bis ins 21. Jahrhundert. Göttingen, $147-175$.

Scheer, G. (2008): Regionalentwicklung und Netzwerke. In: BauerWolf, S.; Payer, H.; Scheer, G. (Hrsg.): Erfolgreich durch Netzwerkkompetenz. Handbuch für Regionalentwicklung. Wien, 1-4.

Schulte, M. (2015): Mehrfachengagierte und ihre Engagementkarrieren. Typen und Einflussfaktoren. Wiesbaden.

Schwarz, C. (1990): Die Landfrauenbewegung in Deutschland. Zur Geschichte einer Frauenorganisation unter besonderer Berücksichtigung der Jahre 1898 bis 1933. Mainz. = Studien zur Volkskultur in Rheinland-Pfalz 9.

Simonson, J.; Vogel, C.; Tesch-Römer, C. (2016): Freiwilliges Engagement in Deutschland. Der Deutsche Freiwilligensurvey 2014. Berlin.

Snelinski, H. (2005): Der LandFrauenverband - Interessenvertretung von auf dem Lande lebenden Frauen - erweist sich als unverzichtbare gestalterische Kraft. In: Rosa-Luxemburg-Stiftung (Hrsg.):
Landfrauen - treibende Kraft der Dorfentwicklung. Dokumentation einer Tagung des Gesprächskreises Ländlicher Raum am 3. Juni 2005 in Berlin. Berlin, 17-21.

Steinführer, A. (2015): Bürger in der Verantwortung. Veränderte Akteursrollen in der Bereitstellung ländlicher Daseinsvorsorge. In: Raumforschung und Raumordnung 73, 1, 5-16.

SVLFG - Sozialversicherung für Landwirtschaft, Forsten und Gartenbau (2013): Kinder lernen gesundes Essen. Pressemitteilung vom 26. Juni 2013. https://www.svlfg.de/63-presse/pres03/03_pres_ pdf 2013/30 2013.pdf (19.05.2017).

von Rohr, G.; Wotha, B. (2006): Motivation und Qualifikation für Aufgaben in der Ländlichen Regionalentwicklung. Hauptamt und Ehrenamt. Studie im Auftrag der Akademie für die Ländlichen Räume Schleswig-Holsteins e. V. (ALR). http://digital.zlb. $\mathrm{de} /$ viewer/content?action=application\&sourcepath=33330986/ Regionalentwicklung.pdf\&format=pdf (19.05.2017).

Walk, H. (2014): Veränderungen lokaler Governance. In: Zimmer, A.; Simsa, R. (Hrsg.): Forschung zu Zivilgesellschaft, NPOs und Engagement: Quo vadis? Wiesbaden, 197-207. = Bürgergesellschaft und Demokratie 46.

Walk, H.; Dienel, H.-L. (2009): Kooperationsnetze und lokale Governance-Formen als Erfolgsfaktoren für ostdeutsche Kommunen. In: Bode, I.; Evers, A.; Klein, A. (Hrsg.): Bürgergesellschaft als Projekt. Wiesbaden, 195-213. = Bürgergesellschaft und Demokratie 28.

Wilde, G. (2014): Zivilgesellschaftsforschung aus Geschlechterperspektive. Zur Ambivalenz von Erweiterung und Begrenzung eines politischen Handlungsraumes. In: Zimmer, A.; Simsa, R. (Hrsg.): Forschung zu Zivilgesellschaft, NPOs und Engagement: Quo vadis? Wiesbaden, 209-227. = Bürgergesellschaft und Demokratie 46.

Zimmer, A. (2007a): Verbände als Dienstleister und Träger öffentlicher Aufgaben. In: von Winter, T.; Willems, U. (Hrsg.): Interessenverbände in Deutschland. Wiesbaden, 393-412.

Zimmer, A. (2007b): Vereine. Zivilgesellschaft konkret. Wiesbaden.

Zimmer, A. (2007c): Vom Ehrenamt zum Bürgerschaftlichen Engagement. Einführung in den Stand der Debatte. In: Schwalb, L.; Walk, H. (Hrsg.): Local Governance - mehr Transparenz und Bürgernähe? Wiesbaden, 95-108.

Zimmer, A. (2009): Zivilgesellschaft und Demokratie. Drei Modelle zivilgesellschaftlicher Einbettung. In: Gesellschaft Wirtschaft Politik 58, 3, 397-406. 